\title{
Quantum kinetic theory of metal clusters in an intense electromagnetic field $I^{*}$
}

\author{
M.Bonitz ${ }^{1,2}$, J.W.Dufty ${ }^{2}$ \\ 1 Institut für Theoretische Physik und Astrophysik, \\ Christian-Albrechts-Universität zu Kiel, \\ Leibnizstr. 15, 24098 Kiel, Germany \\ 2 Department of Physics, University of Florida, \\ Gainesville, FL 32611-8440
}

Received March 30, 2004

\begin{abstract}
A quantum kinetic theory for weakly inhomogeneous charged particle systems is derived within the framework of nonequilibrium Green's functions. The results are of relevance for valence electrons of metal clusters as well as for confined Coulomb systems, such as electrons in quantum dots or ultracold ions in traps and similar systems. To be specific, here we concentrate on the application to metal clusters, but the results are straightforwardly generalized. Therefore, we first give an introduction to the physics of correlated valence electrons of metal clusters in strong electromagnetic fields. After a brief overview on the jellium model and the standard density functional approach to the ground state properties, we focus on the extension of the theory to nonequilibrium. To this end a general gauge-invariant kinetic theory is developed. The results include the equations of motion of the two-time correlation functions, the equation for the Wigner function and an analysis of the spectral function. Here, the concept of an effective quantum potential is introduced which retains the convenient local form of the propagators. This allows us to derive explicit results for the spectral function of electrons in a combined strong electromagnetic field and a weakly inhomogeneous confinement potential.
\end{abstract}

Key words: quantum statistical mechanics, charged clusters, laser-plasma interactions

PACS: 05.30.-d,36.40.Wa,52.38.-r

${ }^{*}$ This paper is devoted to the memory of Yuri L'vovich Klimontovich 


\section{Introduction}

Correlated charged particles are of growing interest in many fields of physics. Here the activities date back to the work on electrolytes and plasmas in the first half of the $20^{\text {th }}$ century, for an overview see [1] and references therein. A systematic kinetic theory for correlated many-body systems was derived from the basic equations of quantum mechanics (von Neumann equation) by Bogolyubov, Born, Green, Kirkwood, Yvon and others, resulting in the famous BBGKY-hierarchy of equations for the nonequilibrium reduced density operators, see [2] for an overview.

A major breakthrough in the theory of nonideal gases and plasmas was achieved by the development of field theoretical methods (second quantization). One branch, represented by Feynman, Schwinger, Martin, Keldysh, Baym, Kadanoff and many others, led to a systematic quantum field theory of plasmas - formulated in terms of Green's functions, e.g. [1,3]. An independent line, pioneered by Klimontovich, focussed on a physically very similar, but formally very different method - Klimontovich's microscopic phase space density, e.g. [4,5]. This approach turned out to be very fruitful for classical nonideal gases and plasmas, whereas for quantum manybody systems, the Green's functions approach appears to be more efficient. The latter, therefore, will be the method of choice in the present paper.

Strong field effects in quantum many-body systems have been successfully incorporated into the Green's functions method by many authors, for an overview and references, cf. [6]. Among the problems of this theory (and similar methods as well) is that the results of many-body approximations (such as gradient expansions) are known to be dependent on the chosen gauge. Therefore, an explicitly gaugeinvariant theory [7] provides a convenient starting point from which approximations can be systematically derived. This method turned out to be fruitful in many fields including semiconductor quantum transport, e.g. [6] and dense plasmas [8,9].

The latter results, however, were limited to spatially homogeneous systems. It is, therefore, of great interest to extend them to the inhomogeneous case. This will allow us to extend the theory to a variety of new systems, including the valence electrons of metal clusters, charged particles in traps, electrons in quantum dots [10] and so on. In this paper, we extend previous work $[8,9]$ to these weakly inhomogeneous systems, concentrating on the additional modifications arising in the quantum kinetic equations from an external confinement field which is the source of the inhomogeneity. For definiteness, we wil consider the potential $V_{I}$ confining the valence electrons in metal clusters, but the situation in quantum dots or particle traps is analogous.

The basic physics of these systems is best illustrated on the jellium approximation (i.e. homogeneous ion charge density within a sphere), as it directly generalizes the very successful one-component plasma model to confined systems. This is done in the reminder of this introductory section and in section 2. As a result of mean-field effects (Hartree-Fock selfenergy effects) this potential is renormalized and becomes density (and possibly time) dependent. The ground state configuration of the valence electrons in this effective potential $V_{I}^{\mathrm{eff}}$ is usually obtained within density functional 
theory. To go beyond the ground state properties and to analyze the nonequilibrium behavior of the electrons in a strong electromagnetic field requires development of a quantum kinetic theory which is outlined in section 3. There, a gauge-invariant derivation is presented which selfconsistently includes the external field and the weakly inhomogeneous confinement potential. The kinetic equations for the twotime correlation functions and for the Wigner distribution are derived.

Special attention is given to an analysis of the spectral properties of the electrons, cf. section 3.9. Introducing the concept of an effective quantum potential which replaces the potential $V_{I}^{\text {eff }}$, the spectral function of the valence electrons in a combined electromagnetic field and thus the confinement potential is derived. Its main characteristic feature is that, even without correlations, the spectral function is broadened and shifted (towards higher energy) as a results of the external field and of inhomogeneity effects. This has direct consequences for various scattering processes and the collision integrals. Further applications of the results to solutions of the quantum kinetic equation in linear response, to dielectric properties and to explicit solutions in a strong field go beyond this paper and will be presented in a forthcoming publication [11].

\subsection{Basic equations and simplifications}

We consider a finite system of $N$ nuclei with atomic mass $A$ and charge $Z$. Thus, the total number of elementary charged particles - nuclei and electrons - is $N(Z+1)$, each interacting by the bare Coulomb potential. The particles are subject to an electromagnetic field given by the vector and scalar potentials $\mathbf{A}(\mathbf{r}, t)$ and $\phi(\mathbf{r}, t)$. The hamiltonian of the full system consists of a field part and a coupled field-matter part,

$$
\begin{aligned}
\hat{H} & =\hat{H}_{\mathrm{F}}+\hat{H}_{\mathrm{FM}}, \\
\hat{H}_{\mathrm{F}} & =\sum_{k, \lambda} \hbar \omega_{k} b_{k \lambda}^{\dagger} b_{k \lambda} \\
\hat{H}_{\mathrm{FM}} & =\hat{H}_{0}+\hat{H}_{\mathrm{int}}, \\
\hat{H}_{0} & =\sum_{\alpha=1}^{N}\left\{\frac{\left(P_{\alpha}-\frac{e_{0}}{c} \mathbf{A}\left(\mathbf{r}_{\alpha}, t\right)\right)^{2}}{2 M}+\phi\left(\mathbf{r}_{\alpha}, t\right)+\sum_{i=1}^{Z} \frac{\left(p_{\alpha i}+\frac{e_{0}}{c} \mathbf{A}\left(\mathbf{r}_{\alpha}, t\right)\right)^{2}}{2 m_{\mathrm{e}}}+\phi\left(\mathbf{r}_{\alpha i}, t\right)\right\}, \\
\hat{H}_{\mathrm{int}} & =\sum_{\alpha=1}^{N} \sum_{\beta=1}^{N}\left\{\frac{1}{2} \frac{\left(Z e_{0}\right)^{2}}{\left|\mathbf{R}_{\alpha}-\mathbf{R}_{\beta}\right|}+\sum_{i=1}^{Z}\left[-\frac{Z e_{0}^{2}}{\left|\mathbf{R}_{\alpha}-\mathbf{r}_{\beta i}\right|}+\sum_{j=1}^{Z} \frac{e_{0}^{2}}{\left|\mathbf{r}_{\alpha i}-\mathbf{r}_{\beta j}\right|}\right]\right\}
\end{aligned}
$$

where $\mathbf{p}$ and $\mathbf{P}$ denote the electron and ion momentum, respectively, $\mathbf{r}$ and $\mathbf{R}$ the corresponding positions and $m_{\mathrm{e}}$ and $M$ the corresponding masses. $e_{0}$ is the free electron charge times minus one, and in $H_{\text {int }}$ selfinteraction terms are not included (first and third terms). $b_{k \lambda}$ and $b_{k \lambda}^{\dagger}$ are annihilation and creation operators of photons of mode $k$ (normally $\mathrm{k}$ denotes the momentum) with polarization $\lambda$ which obey bosonic commutation rules, $\left[b_{k \lambda}, b_{k^{\prime} \lambda^{\prime}}^{\dagger}\right]=\delta_{k k^{\prime}} \delta_{\lambda \lambda^{\prime}}$, all other commutators vanish. The 
transverse vector potential of the electromagnetic field is expressed in terms of these operators by [12]

$$
\hat{\mathbf{A}}(\mathbf{r})=\sum_{k, \lambda}\left(\frac{2 \pi \hbar c^{2}}{\omega_{k} \mathcal{V}}\right)^{1 / 2}\left(b_{k \lambda} \hat{\epsilon}_{k \lambda} \mathrm{e}^{\mathrm{i} \mathbf{k r}}+b_{k \lambda}^{\dagger} \hat{\epsilon}_{k \lambda}^{*} \mathrm{e}^{-\mathrm{ikr}}\right),
$$

where the expansion is in a complete set of plane waves ${ }^{1}$, for convenience, and $\mathcal{V}$ denotes the volume. The transversality condition puts on the constraint $\mathbf{k} \cdot \hat{\epsilon}_{k \lambda}=0$. For sake of generality, we also include a longitudinal electric field which is given by the scalar potential $\phi$. Some issues related to various presentations of the field (gauge problem) will be discussed below.

\subsubsection{Limit of a classical electromagnetic field}

In this paper we are interested specifically in intense electromagnetic field produced by lasers or free electron lasers. These electromagnetic fields are characterized by high coherence and a large number of photons in each mode which justifies a classical treatment. For example, the number of photons in a laser field with intensity $I=1 \mathrm{~W} / \mathrm{cm}^{2}$ and photon energy of $\hbar \omega=1 \mathrm{eV}$ in a typical coherence volume of $\mathcal{V}=1 \mathrm{~cm}^{-3}$ can be estimated by

$$
N_{k \lambda}=\frac{I}{(\hbar \omega)} \frac{\mathcal{V}}{c} \approx 2 \cdot 10^{8} .
$$

This large number assures that fluctuations of the eigenvalues of the photon number operator $\mathbf{n}_{k \lambda}=b_{k \lambda}^{\dagger} b_{k \lambda}$ around its mean value which is given by a classical treatment $\left\langle\mathbf{n}_{k \lambda}\right\rangle \approx N_{k \lambda}$ will be small. The resulting classical result for the vector potential is

$$
\hat{\mathbf{A}}(\mathbf{r}, t) \rightarrow \mathbf{A}(\mathbf{r}, t)=\sum_{k \lambda} \frac{c}{2 \omega_{k}}\left\{\mathbf{E}_{k \lambda} \mathrm{e}^{\mathrm{i}\left(\mathbf{k r}-\omega_{k} t-\phi_{k \lambda}\right)}+\mathbf{E}_{k \lambda}^{*} \mathrm{e}^{-\mathrm{i}\left(\mathbf{k r}-\omega_{k} t-\phi_{k \lambda}\right)}\right\},
$$

where the electric field amplitude is defined by the average (classical) number of photons in the mode, $\mathbf{E}_{k \lambda}=\sqrt{\left(8 \pi \hbar \omega_{k \lambda} N_{k \lambda}\right) / \mathcal{V}} \cdot \hat{\epsilon}_{k \lambda}$, and the $\phi_{k \lambda}$ are operators describing the relative phases of different modes. For a single-mode laser which we will consider below, $\phi_{k \lambda}$ leads just to a time shift and does not enter any physical result and may thus be dropped. For a strict justification of the classical result, see [12].

\subsubsection{Separation of valence electrons}

A first step to simplify the above system is to subdivide the electrons into valence (weakly bound or quasi-free) and core (deeply bound) ones. This is reasonable for

\footnotetext{
${ }^{1}$ This is an operator in the Schrödinger picture. It can be made time-dependent by transforming to the Heisenberg representation by

$$
\mathrm{e}^{\frac{i}{\hbar} \hat{H}_{\mathrm{F}} t} \hat{\mathbf{A}}(\mathbf{r}) \mathrm{e}^{-\frac{i}{\hbar} \hat{H}_{\mathrm{F}} t}=\sum_{k, \lambda}\left(\frac{2 \pi \hbar c^{2}}{\omega_{k} \mathcal{V}}\right)^{1 / 2}\left(b_{k \lambda} \hat{\epsilon}_{k \lambda} \mathrm{e}^{\mathrm{i}\left(\mathbf{k r}-\omega_{k} t\right)}+b_{k \lambda}^{\dagger} \hat{\epsilon}_{k \lambda}^{*} \mathrm{e}^{-\mathrm{i}\left(\mathbf{k r}-\omega_{k} t\right)}\right) .
$$
}


metals and metal clusters and has been found to well reproduce dominant qualitative features, e.g. [13,14]. On the other hand, it has to be kept in mind that this subdivision is never strict and, in situations of strong excitation, it has to be justified in each case. Moreover, in nonequilibrium situations inner core electrons may be ionized or become valence electrons.

Using this idea, the system is reconsidered as the one composed of $N$ ions, each of which is $w$-fold charged ${ }^{2}$, and $w \cdot N$ valence electrons. This means that the field-matter hamiltonian $\hat{H}_{\mathrm{FM}}$ is now replaced by the sum of two terms - an ion hamiltonian and an effective electron hamiltonian, $\hat{H}_{\mathrm{FM}}=\hat{H}_{I}+\hat{H}_{\mathrm{e}}$, given by

$$
\begin{aligned}
\hat{H}_{I} & =\sum_{\alpha=1}^{N}\left\{\frac{\left(P_{\alpha}-\frac{e_{0}}{c} \mathbf{A}\left(\mathbf{r}_{\alpha}, t\right)\right)^{2}}{2 M}+\phi\left(\mathbf{r}_{\alpha}, t\right)+\sum_{\beta<\alpha}^{N} \frac{\left(w e_{0}\right)^{2}}{\left|\mathbf{R}_{\alpha}-\mathbf{R}_{\beta}\right|}\right\}, \\
\hat{H}_{\mathrm{e}} & =\sum_{i=1}^{w N}\left\{\frac{\left(p_{i}+\frac{e_{0}}{c} \mathbf{A}\left(\mathbf{r}_{i}, t\right)\right)^{2}}{2 m_{\mathrm{e}}}+\phi\left(\mathbf{r}_{i}, t\right)+V_{I}\left(\mathbf{r}_{i}, t\right)+\sum_{j<i}^{w} \frac{e_{0}^{2}}{\left|\mathbf{r}_{i}-\mathbf{r}_{j}\right|}\right\}, \\
V_{I}\left(\mathbf{r}_{i}, t\right) & =\sum_{\alpha=1}^{N} V_{\mathrm{ps}}\left(\left|\mathbf{R}_{\alpha}(t)-\mathbf{r}_{i}\right|\right) .
\end{aligned}
$$

The major simplification is that the electron-ion interactions are replaced by an effective single-particle external potential in which the electrons move.

\subsubsection{Adiabatic approximation}

Due to the large ion mass, often the ionic motion can be neglected (it is no principal problem to avoid this approximation and perform a selfconstistent numerical treatment where the ion coordinates are updated on comparatively large time intervals). Since we are interested in fast processes related to the electron dynamics prior to the Coulomb explosion we will use this assumption. At the same time this allows us to neglect the external field effect on the ions, so we will neglect the potentials $\mathbf{A}$ and $\phi$ in the ionic hamiltonian $\hat{H}_{I}$ and, moreover, consider the ions as "frozen". Their only effect on the electrons is then to provide a neutralizing background (which is inhomogeneous though, see section 1.2 below) and the effective potential $V_{I}$. There still remains a time dependence in the potential $V_{I}(t)$ which is due to ionization processes changing the charge of the ions.

\subsection{Spherical jellium model}

In the following we will use atomic units, i.e. lengths will be in units of the hydrogen Bohr radius, $a_{\mathrm{B}}=\hbar^{2} /\left(e_{0}^{2} m_{\mathrm{e}}\right)$ and energies in units of Hartree, $1 \mathrm{Ha}=$ $2 R y=e_{0}^{2} / a_{\mathrm{B}}$. Then, the interparticle distance $a$ is measured by the Wigner-Seitz radius $r_{\mathrm{s}}=a / a_{\mathrm{B}}$.

\footnotetext{
${ }^{2}$ this is analogous to switching from a physical to a chemical picture in partially ionized plasmas, e.g. [1]
} 
The simplest approximation to treat the influence of the ions on the electrons is the spherical jellium model ${ }^{3}$. It is essentially a generalization of the one-component plasma model to the case of finite systems: the ion charge density $\rho_{I}$ is assumed to be homogeneous, inside a sphere of radius $R_{I}$ and zero outside,

$$
\rho_{I}(r)=\rho_{I 0} \Theta\left(R_{I}-r\right), \quad R_{I}=r_{\mathrm{s}} N^{1 / 3} .
$$

Since the number density is assumed to be constant, given by the bulk value $r_{\mathrm{s}}$, the radius of the sphere is determined by the particle number $N$, and the volume of the ion sphere is $\mathcal{V}_{I}=4 \pi r_{\mathrm{s}}^{3} N / 3$. Thus the charge density of the sphere, $\rho_{I 0}$, is given by the charge of all ions inside the sphere,

$$
Q_{I}\left(R_{I}\right)=w N e_{0}: \quad \rho_{I 0}=\frac{Q_{I}\left(R_{I}\right)}{\mathcal{V}_{I}}=\frac{3}{4 \pi} \frac{w e_{0}}{r_{\mathrm{s}}^{3}}
$$

We first compute the electric field of the charged sphere. Outside the sphere, it is just the field of a point charge equal to the total charge, while inside it is the field of a point charge confined in the sphere of radius $r$,

$$
E_{I}(r)= \begin{cases}\frac{w N e_{0}}{r^{2}}, & r>R_{I} \\ \frac{Q_{I}(r)}{r^{2}}=\frac{w e_{0}}{r_{\mathrm{s}}^{3}} r, & r \leqslant R_{I}\end{cases}
$$

since $Q_{I}(r)=\rho_{0 I} 4 \pi r^{3} / 3=w e_{0} r^{3} / r_{\mathrm{s}}^{3}$. From the electric field we immediately obtain the electrostatic potential, given by

$$
\phi_{I}(r)=-\int^{r} \mathrm{~d} \bar{r} E_{I}(\bar{r})+C .
$$

Outside the ion sphere, the potential is $w N e_{0} / r$ and, determining the constant from the continuity of the potential at $r=R_{I}$, we obtain

$$
C=w e_{0}\left[\frac{N}{R_{I}}+\frac{R_{I}^{2}}{2 r_{\mathrm{s}}^{3}}\right]
$$

and

$$
\phi_{I}(r)= \begin{cases}\frac{w N e_{0}}{r}, & r>R_{I} \\ \frac{w e_{0}}{2 r_{\mathrm{s}}^{3}}\left(3 R_{I}^{2}-r^{2}\right), & r \leqslant R_{I} .\end{cases}
$$

Thus, the jellium potential (energy) $V_{I}$ which one electron will feel will be given by $V_{I}(r)=-e_{0} \phi_{I}(r)$,

$$
V_{I}(r)= \begin{cases}-\frac{w N e_{0}^{2}}{r}=-\frac{R_{I}^{3}}{r_{\mathrm{s}}^{3}} \frac{w e_{0}^{2}}{r}, & r>R_{I}, \\ -\frac{w N e_{0}^{2}}{2 R_{I}}\left[3-\left(\frac{r}{R_{I}}\right)^{2}\right]=-\frac{w e_{0}^{2}}{2 r_{\mathrm{s}}^{3}}\left(3 R_{I}^{2}-r^{2}\right), & r \leqslant R_{I} .\end{cases}
$$

\footnotetext{
${ }^{3}$ This model is analogous to the ion sphere model in strongly correlated plasmas which is due to Salpeter [16] and the Wigner-Seitz sphere concept in solid state physics, e.g.[15,17].
} 
Let us briefly discuss the basic properties of the jellium model.

i) By construction, $V_{I}$ is continuous for all $r$ including the sphere boundary $r=R_{I}$. Also, its first derivative, i.e. the force acting on the electrons toward the cluster center, $\mathbf{F}_{I}=-\nabla V_{I}$,

$$
\mathbf{F}_{I}(r)= \begin{cases}-\frac{w N e_{0}^{2}}{r^{3}} \mathbf{r}, & r>R_{I}, \\ -\frac{w N e_{0}^{2}}{R_{I}^{3}} \mathbf{r}, & r \leqslant R_{I},\end{cases}
$$

is continuous at $r=R_{I}$.

ii) The derivative of $F_{I}$ is not continuous. At the point $r=R_{I}, F_{I}^{\prime}(r)$ jumps from $\left(2 w N e_{0}^{2}\right) / R_{I}^{3}$ to $-\left(w N e_{0}^{2}\right) / R_{I}^{3}$. This discontinuity is obvious since $\phi_{I}$ obeys Poisson's equation with the source $\rho_{I}$. In terms of $V_{I}$, the Poisson equation reads $V_{I}^{\prime \prime}(r)=-4 \pi\left(-e_{0}\right) \rho_{I}(r)$ with a discontinuity of $4 \pi e_{0} \rho_{I 0}=\left(3 w N e_{0}^{2}\right) / R_{I}^{3}$ at $r=R_{I}$, in agreement with the above result.

iii) The electrostatic energy contained in the ion sphere is readily computed,

$$
\begin{aligned}
W_{I} & =\frac{1}{2} \int \mathrm{d}^{3} r \rho_{I}(r) \phi_{I}(r)=\frac{4 \pi}{2} \frac{3}{4 \pi} \frac{w e_{0}}{r_{\mathrm{s}}^{3}} \int_{0}^{R_{I}} \mathrm{~d} r r^{2} \frac{w e_{0}}{2 r_{\mathrm{s}}^{3}}\left(3 R_{I}^{2}-r^{2}\right) \\
& =\frac{3}{5} \frac{\left(w N e_{0}\right)^{2}}{R_{I}}
\end{aligned}
$$

iv) Electron binding: the $N_{\text {ve }}$ valence electrons are confined in the potential $V_{I}$. The deepest value of the potential is at the center of the ion sphere,

$$
V_{I}(0)=-\frac{3}{2} \frac{w e_{0}^{2} N^{2 / 3}}{r_{\mathrm{s}}}
$$

We note that this binding energy would refer to a single electron. For many electrons, the Pauli principle leads to occupation of higher energy states between the bottom of the potential, $V_{I}(0)$ and the Fermi energy, see section 2. The spatial distribution of the valence electrons in the potential well is governed by the shape of $V_{I}$ and by quantum and interaction effects among the electrons - i.e. by mean-field, exchange and correlation effects. A simple approach is provided by Thomas-Fermi theory, see section 2 .

As noted above, the potential $V_{I}$ may change in time. In particular, each act of ionization increases the ion charge number $w N \rightarrow w N+1$, thus deepening the potential and increasing the binding.

The potential (10) enters the electron hamiltonian $\hat{H}_{\mathrm{e}}(6)$, replacing the sum over the pseudopotentials $V_{\mathrm{ps}}$. 


\section{Valence electron ground state configuration}

To obtain the ground state of the ensemble of valence electrons one can use the concepts of density functional theory. There one minimizes the total energy with respect to the valence electron number density profile $n(\mathbf{r})$,

$$
\frac{\delta E_{\mathrm{ve}}[n(\mathbf{r})]}{\delta n(\mathbf{r})}=0, \quad \text { with } \int \mathrm{d}^{3} r n(\mathbf{r})=N_{\mathrm{ve}}=\text { const. }
$$

If the cluster is neutral, the total number of electrons in the cloud equals the number of valence electrons $N_{\text {ve }}=w \cdot N$, but for generality we will retain the number $N_{\text {ve }}$. The total energy can be written as

$$
E_{\mathrm{ve}}[n(\mathbf{r})]=T_{\mathrm{ve}}[n(\mathbf{r})]+U_{\mathrm{ve}}^{\mathrm{H}}[n(\mathbf{r})]+V_{\mathrm{ve}}[n(\mathbf{r})]+E_{\mathrm{ve}}^{\mathrm{XC}}[n(\mathbf{r})],
$$

consisting of kinetic energy (first term), Hartree mean field (second), external confinement (third) and a remainder (fourth) containing all exchange and correlation contributions. The quality of the result depends on the approximation for the various energy contributions.

\subsection{Thomas-Fermi model}

An illustrative nontrivial approximation for the total energy of the valence electrons is to include the electron kinetic energy, the external confinement $V_{I}$ in the jellium approximation and the electron mean field (Hartree potential) ${ }^{4}$,

$$
E_{\mathrm{ve}}[n(\mathbf{r})]=T_{\mathrm{ve}}[n(\mathbf{r})]+U_{\mathrm{ve}}^{\mathrm{H}}[n(\mathbf{r})]+V_{\mathrm{ve}}[n(\mathbf{r})] .
$$

The kinetic energy may be approximated by the Thomas-Fermi result, i.e. one assumes that the valence electrons are governed by a zero-temperature Fermi distribution which parametrically depends on a space-dependent density $n(\mathbf{r})$, (local approximation, LDA). With the kinetic energy density of the ideal electron gas,

$$
\epsilon=\frac{3}{10} \frac{\hbar^{2}\left(3 \pi^{2}\right)^{2 / 3}}{m_{\mathrm{e}}} n^{5 / 3}
$$

we obtain

$$
T_{\mathrm{ve}}[n(\mathbf{r})]=\frac{3}{10} \frac{\hbar^{2}\left(3 \pi^{2}\right)^{2 / 3}}{m_{\mathrm{e}}} \int \mathrm{d}^{3} r n^{5 / 3}(\mathbf{r})
$$

Next, the Hartree mean-field energy is given by

$$
\begin{aligned}
U_{\mathrm{ve}}^{\mathrm{H}} & =\frac{e_{0}^{2}}{2} \int \mathrm{d}^{3} r \mathrm{~d}^{3} r^{\prime} \frac{n(\mathbf{r}) n\left(\mathbf{r}^{\prime}\right)}{\left|\mathbf{r}-\mathbf{r}^{\prime}\right|}=\frac{1}{2} \int \mathrm{d}^{3} r n(\mathbf{r}) \Sigma_{\mathrm{ve}}^{\mathrm{H}}[\mathbf{r} ; n(\mathbf{r})], \\
\Sigma_{\mathrm{ve}}^{\mathrm{H}}[\mathbf{r} ; n(\mathbf{r})] & =e_{0}^{2} \int \mathrm{d}^{3} r^{\prime} \frac{n\left(\mathbf{r}^{\prime}\right)}{\left|\mathbf{r}-\mathbf{r}^{\prime}\right|}
\end{aligned}
$$

\footnotetext{
${ }^{4}$ thus neglecting in equation (14) the contributions $E^{\mathrm{XC}}$ from electron exchange (Fock energy) and correlations.
} 
where we introduced the Hartree selfenergy of the valence electrons, and we note that it is the solution of Poisson's equation

$$
\nabla^{2} \Sigma_{\mathrm{ve}}^{\mathrm{H}}[\mathbf{r} ; n(\mathbf{r})]=-4 \pi e_{0}^{2} n(\mathbf{r}) .
$$

Finally, the confinement energy of the valence electrons in the jellium potential is given by the integral of the ion electrostatic potential multiplied with the electron charge density,

$$
V_{\text {ve }}[n(\mathbf{r})]=-e_{0} \int \mathrm{d}^{3} r \phi_{I}(r) n(\mathbf{r})=\int \mathrm{d}^{3} r V_{I}(r) n(\mathbf{r}) .
$$

Collecting the results for the three energy contributions, we can perform the variation of the total energy (15) with respect to the valence electron density profile

$$
\delta E=\int \mathrm{d}^{3} r\left\{\frac{\hbar^{2}\left(3 \pi^{2}\right)^{2 / 3}}{2 m_{\mathrm{e}}} n^{2 / 3}(\mathbf{r})+e_{0}^{2} \int \mathrm{d}^{3} r^{\prime} \frac{n\left(\mathbf{r}^{\prime}\right)}{\left|\mathbf{r}-\mathbf{r}^{\prime}\right|}+V_{I}(r)-\mu\right\} \delta n(\mathbf{r}),
$$

where we introduced the chemical potential as Lagrange multiplier. Requiring the expression in curly brackets to vanish, we obtain

$$
n(\mathbf{r})=\frac{1}{3 \pi^{2} \hbar^{3}}\left\{2 m_{\mathrm{e}}\left[\left|V_{I}(r)\right|-\Sigma_{\mathrm{ve}}^{\mathrm{H}}[\mathbf{r} ; n(\mathbf{r})]+\mu\right]\right\}^{3 / 2} .
$$

This is not an explicit equation for the density because the Hartree term depends on $n$ too. We can recast it into a closed differential equation for the Hartree selfenergy by inserting (22) into the r.h.s. of the Poisson equation (19),

$$
\nabla^{2} \Sigma_{\mathrm{ve}}^{\mathrm{H}}[\mathbf{r} ; n(\mathbf{r})]=-\frac{4 e_{0}^{2}}{3 \pi \hbar^{3}}\left\{2 m_{\mathrm{e}}\left[\left|V_{I}(r)\right|-\Sigma_{\mathrm{ve}}^{\mathrm{H}}[\mathbf{r} ; n(\mathbf{r})]+\mu\right]\right\}^{3 / 2} .
$$

We will concentrate below on the jellium potential $V_{I}$. Due to its isotropy, the valence electron density will be isotropic as well. Then, from equation (22), there are two obvious constraints on the density $n(r)$ : since $V_{I}$ and $\Sigma_{\mathrm{ve}}^{\mathrm{H}}$ are monotonically decaying with increasing distance from the $\operatorname{center}^{5}, n(r)$ will be monotonically decaying too, reaching zero at a finite $r_{0}$ and remain zero for $r>r_{0}$. The critical radius $r_{0}$ follows from the normalization,

$$
4 \pi \int_{0}^{r_{0}} \mathrm{~d} r r^{2} n(r)=N_{\mathrm{ve}}
$$

and defines the chemical potential:

$$
\mu=-\left|V_{I}\left(r_{0}\right)\right|+\Sigma_{\mathrm{ve}}^{\mathrm{H}}\left[r_{0} ; n(r)\right] .
$$

\footnotetext{
${ }^{5}$ the latter statement has to be verified on the final solution for the density
} 
The angle integration in the selfenergy (18) can be carried out ${ }^{6}$

$$
\begin{gathered}
\Sigma_{\mathrm{ve}}^{\mathrm{H}}[r ; n(r)]=4 \pi e_{0}^{2} \int_{0}^{r_{0}} \mathrm{~d} r^{\prime} r^{\prime 2} n\left(r^{\prime}\right) g\left(r, r^{\prime}\right), \\
\text { with } g\left(r, r^{\prime}\right)= \begin{cases}\frac{1}{r}, & r>r^{\prime}, \\
\frac{1}{r^{\prime}}, & r \leqslant r^{\prime} .\end{cases}
\end{gathered}
$$

In particular, for $r \geqslant r_{0}: \Sigma_{\mathrm{ve}}^{\mathrm{H}}(r)=\frac{N_{\mathrm{ve}} e_{0}^{2}}{r}$. Using this result at $r=r_{0}$ and expression (10) for the jellium potential ${ }^{7}$ we obtain from (25)

$$
\mu=-\left(w N-N_{\mathrm{ve}}\right) \frac{e_{0}^{2}}{r_{0}} .
$$

We see that for a neutral cluster, the chemical potential vanishes, in agreement with atomic Thomas-Fermi theory, e.g. [18]. For positively charged clusters where some electrons have been removed from the cloud, $N_{\text {ve }}<w N, \mu$ is negative.

Equations (22), (24), (28) provide a closed system to compute the valence electron density profile, the cloud radius $r_{0}$, the chemical potential and the Fermi energy, $E_{\mathrm{F}}=-\mu$, of the valence electrons surrounding the jellium droplet.

\subsection{Free Electron gas}

The simplest limiting case is the ideal electron gas where the Hartree mean field is neglected as well. This case is instructive as all results can be obtained analytically. Moreover, this limit serves as a generalization of the well-known model of the (macroscopic) ideal electron gas to finite systems, and it becomes exact in the high-density limit, $r_{\mathrm{s}} \rightarrow 0$. Then we obtain from equations (22), (28)

$$
\begin{aligned}
& \mu^{i d}=-\frac{w N e_{0}^{2}}{r_{0}}, \\
& n^{i d}(r)=\left\{\begin{array}{lr}
\frac{\left(2 m_{\mathrm{e}}\right)^{3 / 2}}{3 \pi^{2} \hbar^{3}}\left(\frac{w N e_{0}^{2}}{2 R_{I}}\right)^{3 / 2}\left[3-\left(\frac{r}{R_{I}}\right)^{2}-2 \frac{R_{I}}{r_{0}}\right]^{3 / 2}, & r \leqslant R_{I}=r_{\mathrm{s}} N^{1 / 3} ; \\
\frac{\left(2 m_{\mathrm{e}}\right)^{3 / 2}}{3 \pi^{2} \hbar^{3}}\left(\frac{w N e_{0}^{2}}{r}\right)^{3 / 2}\left[1-\frac{r}{r_{0}}\right]^{3 / 2}, & R_{I} \leqslant r \leqslant r_{0} ; \\
0, & r>r_{0} .
\end{array}\right.
\end{aligned}
$$

${ }^{6}$ due to isotropy,

$$
\begin{aligned}
\int \mathrm{d}^{3} r^{\prime} \frac{n\left(r^{\prime}\right)}{\left|\mathbf{r}-\mathbf{r}^{\prime}\right|} & =2 \pi \int_{0}^{\infty} \mathrm{d} r^{\prime} r^{\prime 2} \int_{-1}^{1} \mathrm{~d} z \frac{n\left(r^{\prime}\right)}{\sqrt{r^{2}+r^{\prime 2}-2 r r^{\prime} z}} \\
& =\frac{2 \pi}{r} \int_{0}^{\infty} \mathrm{d} r^{\prime} r^{\prime}\left\{\sqrt{r^{2}+r^{\prime 2}+2 r r^{\prime}}-\sqrt{r^{2}+r^{\prime 2}-2 r r^{\prime}}\right\}
\end{aligned}
$$

leading to the result (2.1).

${ }^{7}$ The point $r_{0}$ is expected to be outside the ion sphere, so we use the branch of $V_{I}$ which corresponds to $r>R_{I}$. 
Finally, the boundary of the electron cloud, $r_{0}$, follows from equation (24) where the integration can be performed yielding a transcendental equation for $x_{0}=r_{0} / R_{I}$ which, however, is rather complicated ${ }^{8}$, so a numerical solution of equation (24) is advantageous.



$\mathrm{Na}_{20}^{2+}$

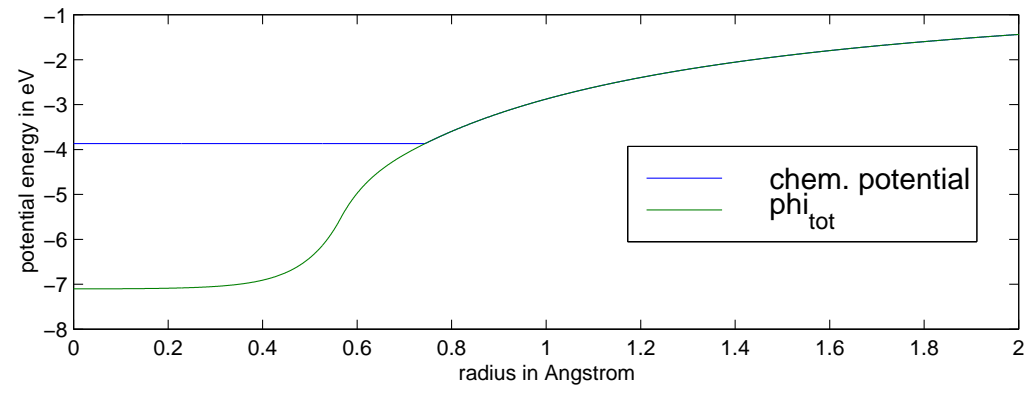

Figure 1. Jellium model result for a two-fold charged sodium cluster of 20 atoms in the Hartree approximation. Upper figure: density of ions and valence electrons vanishing at $R_{I}$ and $r_{0}$, respectively. Lower figure: total potential including $V_{I}$ and the Hartree mean field. Also shown is the chemical potential. Its intersection with the total potential defines the electron cloud boundary $r_{0}$. Figure courtesy of Th. Fennel.

\footnotetext{
${ }^{8}$ for completeness, we provide the result of the integration using equation (30) for the density profile. Introducing the definitions $n \equiv \frac{n}{n^{i d}}, x_{0} \equiv \frac{r_{0}}{R_{I}}, \alpha \equiv \frac{N_{\mathrm{ve}}}{w N}, n_{0} \equiv \frac{\left(2 m_{\mathrm{e}}\right)^{3 / 2}}{3 \pi^{2} \hbar^{3}}\left(\frac{w N e_{0}^{2}}{2 R_{I}}\right)^{3 / 2}, x_{0}$ is the solution of the following equation:

$$
\begin{aligned}
\frac{N_{\mathrm{ve}}}{4 \pi R_{I}^{3} n_{0}} & =-\frac{\left(2 \alpha-x_{0}\right)^{3}}{16 x_{0}^{3}} \operatorname{arccot}\left[\sqrt{2-\frac{2 \alpha}{x_{0}}}\right]-\frac{\sqrt{2-\frac{2 \alpha}{x_{0}}}}{48 x_{0}^{2}}\left(6 \alpha-7 x_{0}\right)\left(2 \alpha+x_{0}\right) \\
& +\left(\frac{x_{0}}{2 \alpha}\right)^{3}\left\{\arctan \left[\sqrt{\frac{\alpha}{1-\alpha}}\right]-\operatorname{arccot}\left[\sqrt{\frac{\mathrm{x}_{0}}{\alpha}-1}\right]\right\} \\
& -\frac{1}{24 \alpha^{2}}\left\{(2 \alpha-3)(4 \alpha-1) x_{0}^{3} \sqrt{\frac{1-\alpha}{\alpha}}-\left(2 \alpha-3 x_{0}\right)\left(4 \alpha-x_{0}\right) x_{0}^{3} \sqrt{\frac{x_{0}}{\alpha}-1}\right\} .
\end{aligned}
$$
}




\subsection{Electron ground state including the mean field}

Using the result for the ideal electron gas, we can now improve the valence electron density by including the Hartree mean field. A simple perturbation approach consists in computing $\Sigma^{\mathrm{H}}$, equation (2.1), using $n^{i d}$ and inserting the result into (22) to obtain an improved approximation for the density profile. This procedure can be repeated (at each step, the cut-off radius $r_{0}$ has to be computed again) until convergence is reached.

Numerical results for the electron density in the Hartree approximation are shown in figure 1. One sees that the valence electron density (upper figure) extends beyond the limits of the core ion distribution up to the maximum radius $r_{0}$ which is defined by the intersection of the chemical potential with the effective potential, cf. equation (25). The effective potential deviates from the quadratic (inside the cluster) jellium potential, equation (10) - the mean field effects cause a flat potential shape in the center of the cluster. At the boundary of the ion charge the potential curvature changes turning to an $1 / r$ decay which is only weakly influenced by many-particle effects.

\subsection{Electron ground state including exchange in local approximation}

The mean field result shown in figure 1 is readily improved by including exchange effects. The exchange energy in local approximation was derived by Dirac [19]

$$
E^{\mathrm{X}}[n(\mathbf{r})]=-\frac{3}{4}\left(\frac{3}{\pi}\right)^{1 / 3} e_{0}^{2} \int \mathrm{d}^{3} r[n(\mathbf{r})]^{4 / 3},
$$

and its variation yields the exchange potential

$$
V^{\mathrm{X}}[n(\mathbf{r})]=-\frac{e_{0}^{2}}{\pi}\left(3 \pi^{2}\right)^{1 / 3} \int \mathrm{d}^{3} r[n(\mathbf{r})]^{1 / 3},
$$

which has to be added to the density equation (22). For our isotropic jellium problem this equation then becomes

$$
n(\mathbf{r})=\frac{1}{3 \pi^{2} \hbar^{3}}\left\{2 m_{\mathrm{e}}\left[\left|V_{I}(r)\right|-\Sigma_{\mathrm{ve}}^{\mathrm{H}}[r ; n(r)]+V^{\mathrm{X}}[n(r)]-\left(w N-N_{\mathrm{ve}}\right) \frac{e_{0}^{2}}{r_{0}}\right]\right\}^{3 / 2}
$$

where we used the solution (28) for the chemical potential.

Summarizing this introductory part, we have given a brief overview on the ground state properties of valence electrons in metal clusters. An illustrative picture is given by the Thomas-Fermi model which is generalized by the concepts of density functional theory by including mean field and exchange (plus correlations, in some approximation). The DFT approach is very successful in describing the ground state of clusters, and it allows to systematically improve the model. However, it is not able to correctly describe clusters at elevated temperatures and in nonequilibrium, in particular, under the combined influence of strong fields and nontrivial electronic correlations. 
We now proceed to a rigorous and consistent theoretical description of nonequilibrium properties of metal clusters in a strong electromagnetic field, based on quantum kinetic theory.

\section{Quantum kinetic description of cluster valence electrons in a strong electromagnetic field}

In the previous section we have established the model for our treatment of the valence electrons in metal clusters: it is based on the hamiltonian $\hat{H}_{\mathrm{e}}$, equation (6). The ions are treated as frozen (adiabatic approximation), they have a homogeneous density inside the droplet of radius $R_{I}$, their influence on the electrons is condensed in the jellium potential $V_{I}$. In the absence of an external field, the electrons will form a cloud around the ionic core with a density profile $n(r)$, decreasing away from the center, cf. figure 1, (except for possible Friedel oscillations). This spatial density profile has been computed for the zero temperature limit. At the same time, in momentum space, the electrons will be described by a Fermi distribution (step function) where all momentum states are occupied up to the Fermi energy of the electron cloud, given by minus the chemical potential (28), which is essentially defined by the overall charge of the cluster.

Of course, at finite temperature and, more generally, under nonequilibrium conditions created by a strong field, the momentum distribution of the valence electrons may be very far from the zero-temperature result. To outline a theory which allows to systematically calculate this nonequilibrium distribution and the related transport quantities of the valence electrons is the main subject of this Section.

\subsection{Outline of the presented theory}

While a quantum kinetic theory can be formulated for arbitrary spatial properties, in a weakly inhomogeneous system the formulation strongly simplifies. Moreover, it is reasonable to expect that, under a number of relevant conditions, the spatial shape of the electron cloud will remain close the ground state case, at least for an intermediate period of time prior to the Coulomb explosion. In particular, such a situation is expected to exist, even in nonequilibrium, in the case of

a. homogeneous laser excitation, i.e. if the laser focus and the wave length are much larger than the cluster - which is usually fulfilled, at least in the optical an UV range

b. collective effects influencing the electron cloud as a whole, which is the case for plasma oscillations with sufficiently small wave number, $k \ll 2 \pi / r_{0}$, where $r_{0}$ denotes the extension of the valence electron cloud discussed above,

c. correlation and scattering effects being sufficiently weak.

If these conditions are fulfilled we may expect that, on the scale of the cluster, spatial variations and spatial density gradients will be small (compared to the unperturbed 
density profile), and the main effect of the excitation will be a deformation of the momentum distribution of the electrons. Under these conditions the quantum kinetic theory is effectively formulated in the Wigner representation. Assuming throughout this paper weak spatial inhomogeneity we expect that the local approximation plus first order gradient corrections will give the dominant contributions to the theory. Finally, since gradient corrections are gauge dependent, we will develop a theory which is gauge-invariant from the beginning.

\subsection{Valence Electron Density Matrix}

The central quantity to describe the statistical properties of the electrons in nonequilibrium is the single-particle density matrix $F_{1}\left(\mathbf{r}_{1}, \mathbf{r}_{1}^{\prime}, t\right)$. It can be derived from the von Neumann equation which includes the electron hamiltonian (6)

$$
\mathrm{i} \hbar \frac{\partial}{\partial t} \rho_{\mathrm{e}}-\left[H_{\mathrm{e}}, \rho_{\mathrm{e}}\right]=0
$$

where $\rho_{\mathrm{e}}$ is the density matrix of all $N_{\text {ve }}$ valence electrons. Computing the partial trace of $\rho_{\mathrm{e}}$ over particles $2, \ldots N_{\text {ve }}$ yields the single-particle density operator $F_{1}$ which obeys the first equation of the BBGKY hierarchy, e.g. [2]. In coordinate representation it becomes the one-particle density matrix $F_{1}\left(\mathbf{r}_{1}, \mathbf{r}_{1}^{\prime}, t\right)$. In the following, it will be convenient to transform to center of mass $(\mathbf{R})$ and relative $(\mathbf{r})$ coordinates, and to proceed analogously with time and momentum arguments, according to the relations

$$
\begin{aligned}
\mathbf{R} & \equiv\left(\mathbf{r}_{1}+\mathbf{r}_{1}^{\prime}\right) / 2, \quad \mathbf{r} \equiv \mathbf{r}_{1}-\mathbf{r}_{1}^{\prime}, \\
\mathbf{Q} & \equiv\left(\mathbf{k}_{1}+\mathbf{k}_{1}^{\prime}\right) / 2, \quad \mathbf{k} \equiv \mathbf{k}_{1}-\mathbf{k}_{1}^{\prime}, \\
t & \equiv\left(t_{1}+t_{1}^{\prime}\right) / 2, \quad \tau \equiv t_{1}-t_{1}^{\prime} .
\end{aligned}
$$

In the new coordinates, the density matrix becomes $F_{1}(\mathbf{R}+\mathbf{r} / 2, \mathbf{R}-\mathbf{r} / 2, t) \equiv$ $\tilde{F}_{1}(\mathbf{R}, \mathbf{r}, t)$. Fourier transform with respect to $\mathbf{r}$ then yields the Wigner distribution function $f(\mathbf{R}, \mathbf{p}, t)$, which is analogous to the classical distribution function (however, in contrast to the latter it has the known problems of possible negative and complex values resulting from the Heisenberg uncertainty principle).

Yet, there are a number of nontrivial questions concerning the equation of motion for this function in the special situation of interest which are related to the appearance of the time-dependent field, the spatial inhomogeneity of the cluster etc. For this reason, we turn now to a careful derivation of this equation. In particular, we will use a gauge-invariant approach to exclude any ambiguity of the results. The most straightforward approach to do this is to use nonequilibrium Green's functions as was demonstrated for a homogeneous system in $[8,9]$ which we will generalize to inhomogeneous systems in section 3.6.

\subsection{Local approximation in nonequilibrium and first order gradient correc- tions}

As a first approximation to the rather complicated problem of the valence electron dynamics we introduce a local approximation, in analogy to the concepts used 
in density functional theory ${ }^{9}$. In situations where no sharp spatial changes of the system properties occur (geometrical boundaries etc.) and the number of electrons involved is not small, it is reasonable to assume that the density matrices depend on space (center of mass coordinate) only via the density,

$$
\begin{aligned}
\tilde{F}_{1}(\mathbf{R}, \mathbf{r}, t) & \equiv \tilde{F}_{1}[n(\mathbf{R}, t), \mathbf{r}, t], \\
\text { and, therefore, } \quad \nabla_{R} \tilde{F}_{1}(\mathbf{R}, \mathbf{r}, t) & =\frac{\delta \tilde{F}_{1}}{\delta n(\mathbf{R}, t)} \nabla_{R} n(\mathbf{R}, t),
\end{aligned}
$$

and, similarly for the Wigner function,

$$
\begin{aligned}
f(\mathbf{R}, \mathbf{k}, t) & \equiv f[n(\mathbf{R}, t), \mathbf{k}, t], \\
\text { and, therefore, } \quad \nabla_{R} f(\mathbf{R}, \mathbf{k}, t) & =\frac{\delta f}{\delta n(\mathbf{R}, t)} \nabla_{R} n(\mathbf{R}, t) .
\end{aligned}
$$

Analogous behavior is expected for other dynamical quantities, including two-particle and higher order distributions and the two-time correlation functions, see below.

The situation is more complex for more general quantities which are, for example, a product of several distribution functions. Then, applying the local approximation to each factor does not necessarily result in an adequate approximation for the product. Consider an arbitrary physical quantity $C$ which is the product of $A$ and $B$. In the Wigner representation, we can write (time arguments appear here only as parameters and are omitted),

$$
\begin{aligned}
C(\mathbf{R}, \mathbf{k})= & \int \frac{\mathrm{d}^{3} Q_{1}}{(2 \pi \hbar)^{3}} \int \frac{\mathrm{d}^{3} Q_{2}}{(2 \pi \hbar)^{3}} \int \mathrm{d}^{3} r_{1} \int \mathrm{d}^{3} r_{2} \mathrm{e}^{\frac{i}{\hbar}\left(\mathbf{k}-\mathbf{Q}_{2}\right) \mathbf{r}_{1}} \mathrm{e}^{-\frac{i}{\hbar}\left(\mathbf{k}-\mathbf{Q}_{1}\right) \mathbf{r}_{2}} \\
& \times A\left(\mathbf{R}+\frac{\mathbf{r}_{1}}{2}, \mathbf{Q}_{1}\right) B\left(\mathbf{R}+\frac{\mathbf{r}_{2}}{2}, \mathbf{Q}_{2}\right)
\end{aligned}
$$

which is still general for an arbitrary inhomogeneous case, cf. e.g. [30]. Now, expanding $A$ and $B$ with respect to small deviations of the coordinates with respect to $\mathbf{R}$, up to first order, yields

$$
\begin{aligned}
& A\left(\mathbf{R}+\frac{\mathbf{r}_{1}}{2}\right) B\left(\mathbf{R}+\frac{\mathbf{r}_{2}}{2}\right) \approx A(\mathbf{R}) B(\mathbf{R}) \\
& \quad+\left.\frac{1}{2}\left\{\left[\mathbf{r}_{1} \cdot \nabla_{\mathbf{R}_{1}}+\mathbf{r}_{2} \cdot \nabla_{\mathbf{R}_{2}}\right] A\left(\mathbf{R}_{1}\right) B\left(\mathbf{R}_{2}\right)\right\}\right|_{\mathbf{R}_{1}=\mathbf{R}_{2}=\mathbf{R}} .
\end{aligned}
$$

Using in equation (43) the identities $\mathbf{r}_{1}=-\hbar / \mathrm{i} \nabla_{\mathbf{Q}_{2}}$ and $\mathbf{r}_{2}=\hbar / \mathrm{i} \nabla_{\mathbf{Q}_{1}}$ with the expansion (44) allows to perform the integrations over $\mathbf{r}_{1}$ and $\mathbf{r}_{2}$ and, with the help of the resulting delta functions, also the integrations over $\mathbf{Q}_{1}$ and $\mathbf{Q}_{1}$, yielding $\mathbf{Q}_{1}=\mathbf{Q}_{2}=\mathbf{k}$, with the final result

$$
\begin{aligned}
C(\mathbf{R}, \mathbf{k}) & =\left.\left\{\left[1+\frac{\mathrm{i} \hbar}{2}\left(\nabla_{\mathbf{R}_{1}} \nabla_{\mathbf{Q}_{2}}-\nabla_{\mathbf{R}_{2}} \nabla_{\mathbf{Q}_{1}}\right)\right] A\left(\mathbf{R}_{1}, \mathbf{Q}_{1}\right) B\left(\mathbf{R}_{2}, \mathbf{Q}_{2}\right)\right\}\right|_{(\mathbf{R}, \mathbf{k})} \\
& \equiv\left(1+\frac{i \hbar}{2}\{\ldots\}\right) A B,
\end{aligned}
$$

\footnotetext{
${ }^{9}$ and similar (and even more general) concepts in hydrodynamics
} 
where we introduced the short notation $(\mathbf{R}, \mathbf{k})$ to indicate that, after differentiation, one has to set $\mathbf{R}_{1}=\mathbf{R}_{2}=\mathbf{R}$ and $\mathbf{Q}_{1}=\mathbf{Q}_{2}=\mathbf{k}$. The first term in equation (45) gives the local approximation for $C$, while the term in curly brackets (Poisson brackets) contains the first gradient corrections.

With this general result which will be used below, e.g. for the collision integrals, it is straightforward to test, in each case of interest, the validity of the local approximation and to estimate the magnitude of corrections. On the other hand, it turns out that several physical contributions to the Wigner function, such as the exchange self energy, are missing in the local approximation and appear only in first order gradient corrections, see section 3.7 .

\subsection{Kadanoff-Baym/Keldysh equations for the valence electrons}

The field theoretical description ${ }^{10}$ of the cluster valence electrons is based on the fermionic creation and annihilation operators $\psi^{\dagger}$ and $\psi$ which are defined to guarantee the spin statistics theorem,

$$
\begin{aligned}
\psi(1) \psi(2)+\psi(2) \psi(1) & =\psi^{\dagger}(1) \psi^{\dagger}(2)+\psi^{\dagger}(2) \psi^{\dagger}(1)=0, \\
\psi(1) \psi^{\dagger}(2)+\psi^{\dagger}(2) \psi(1) & =\delta(1-2),
\end{aligned}
$$

where $t_{1}=t_{2}$ has been assumed, and $1 \equiv\left(\mathbf{r}_{1}, t_{1}, s_{1}^{3}\right)$, (below, we will drop the spin index). The nonequilibrium state of the electrons is described by the two-time correlation functions which are statistical averages (with the initial density operator of the system) of field operator products

$$
g^{>}\left(1,1^{\prime}\right)=\frac{1}{\mathrm{i} \hbar}\left\langle\psi(1) \psi^{\dagger}\left(1^{\prime}\right)\right\rangle, \quad g^{<}\left(1,1^{\prime}\right)=-\frac{1}{\mathrm{i} \hbar}\left\langle\psi^{\dagger}\left(1^{\prime}\right) \psi(1)\right\rangle,
$$

where $g^{>}$and $g^{<}$are, in nonequilibrium, independent from one another. They contain the complete dynamical and statistical information. The latter follows from their elements along the time diagonal: the one-particle density matrix is immediately obtained from the function $g^{<}$according to

$$
F_{1}\left(\mathbf{r}_{1}, \mathbf{r}_{1}^{\prime}, t\right)=-\left.\mathrm{i} \hbar g^{<}\left(1,1^{\prime}\right)\right|_{t_{1}=t_{1}^{\prime}}
$$

whereas the dynamical information (e.g. the single-particle spectrum and the correlations) follows from the function values across the diagonal in the $t_{1}-t_{1}^{\prime}$-plane, in particular, from the spectral function $a\left(1,1^{\prime}\right)$,

$$
a\left(1,1^{\prime}\right) \equiv \mathrm{i} \hbar\left\{g^{>}\left(1,1^{\prime}\right)-g^{<}\left(1,1^{\prime}\right)\right\}=\mathrm{i} \hbar\left\{g^{R}\left(1,1^{\prime}\right)-g^{A}\left(1,1^{\prime}\right)\right\},
$$

where $g^{R / A}$ are the retarded and advanced Green's functions, defined below in equation (52). In particular, in cases where the microscopic variables vary on much smaller scales than the macroscopic ones, cf. definition $(36,38)$, it is advantageous

\footnotetext{
${ }^{10}$ In this section we follow [9].
} 
to perform a Fourier transformation with respect to $\tau$ and/or $\mathbf{r}$ which leads to the frequency and momentum variables $\omega$ and p, respectively. In particular, equation (47) then yields the familiar Wigner distribution function

$$
f(\mathbf{p}, \mathbf{R}, t)=-\left.\mathrm{i} \hbar g^{<}\left(\mathbf{p}, \mathbf{R} ; t_{1}, t_{1}^{\prime}\right)\right|_{t_{1}=t_{1}^{\prime}=t} .
$$

The time evolution of the correlation functions in an electromagnetic field is determined by the Kadanoff-Baym/Keldysh equations which follow from the Heisenberg equations of motion for the field operators in the presence of the hamiltonian $\hat{H}_{\mathrm{e}}$ (in the following, the electrostatic potential $\phi$ will be neglected which will be justified in section 3.5)

$$
\begin{aligned}
{[\mathrm{i} \hbar} & \left.\frac{\partial}{\partial t_{1}}-\frac{1}{2 m_{\mathrm{e}}}\left(\frac{\hbar}{\mathrm{i}} \nabla_{1}+\frac{e_{0}}{c} \mathbf{A}(1)\right)^{2}-V_{I}(1)\right] g^{\gtrless}\left(1,1^{\prime}\right)= \\
= & \int \mathrm{d} \overline{\mathbf{r}}_{1} \Sigma^{\mathrm{HF}}\left(1, \overline{\mathbf{r}}_{1} t_{1}\right) g^{\gtrless}\left(\overline{\mathbf{r}}_{1} t_{1}, 1^{\prime}\right)+\int_{t_{0}}^{t_{1}} \mathrm{~d} \overline{1}\left[\Sigma^{>}(1, \overline{1})-\Sigma^{<}(1, \overline{1})\right] g^{\gtrless}\left(\overline{1}, 1^{\prime}\right) \\
& -\int_{t_{0}}^{t_{1}^{\prime}} \mathrm{d} \overline{1} \Sigma^{\gtrless}(1, \overline{1})\left[g^{>}\left(\overline{1}, 1^{\prime}\right)-g^{<}\left(\overline{1}, 1^{\prime}\right)\right],
\end{aligned}
$$

which have to be fulfilled together with the adjoint equations. The 1.h.s. of this equation contains all single-particle terms, whereas the r.h.s. contains all corrections due to mean field, exchange and correlations. Further, $t_{0}$ denotes the initial time where the system is assumed to be uncorrelated (otherwise, the equations have to be supplemented with an initial correlation contribution to $\Sigma$, cf. [20,21]). $\Sigma^{\mathrm{HF}}$ is the Hartree-Fock selfenergy (Hartree mean-field plus exchange energy),

$$
\Sigma^{\mathrm{HF}}\left(11^{\prime}\right)=-\mathrm{i} \hbar \delta\left(t_{1}-t_{1^{\prime}}\right)\left\{\int \mathrm{d} \mathbf{r}_{2} V\left(\mathbf{r}_{1}-\mathbf{r}_{2}\right) g^{<}\left(22^{+}\right)-V\left(\mathbf{r}_{1}-\mathbf{r}_{1}^{\prime}\right) g^{<}\left(11^{\prime}\right)\right\}
$$

and $\Sigma \gtrless$ are the correlation selfenergies which describe collision processes, ionization and so on.

The properties of the cluster valence electrons are mainly determined by the choice of the confinement potential and the collision integrals (selfenergies). In the derivations below, the potential $V_{I}$ will be kept completely general and it will not necessarily be identified with the smooth jellium potential of section 2. Further we may account for varying valence electron number due to ionization of core electrons (or, vice versa, recombination) as well as due to "evaporation" of electrons. These processes can be readily incorporated into the model by choosing the appropriate selfenergy contributions and by properly updating the confinement potential so that it is consistent with the actual number of core and valence electrons.

For the following derivations, it is useful to introduce, in addition to the two-time correlation functions, the retarded and advanced Green's functions

$$
g^{R / A}\left(1,1^{\prime}\right)= \pm \Theta\left[ \pm\left(t_{1}-t_{1}^{\prime}\right)\right]\left\{g^{>}\left(1,1^{\prime}\right)-g^{<}\left(1,1^{\prime}\right)\right\}
$$


which are related by

$$
\left[g^{R}\left(1,1^{\prime}\right)\right]^{*}=g^{A}\left(1^{\prime}, 1\right),
$$

and obey the simpler equations

$$
\begin{aligned}
& {\left[\mathrm{i} \hbar \frac{\partial}{\partial t_{1}}-\frac{1}{2 m_{\mathrm{e}}}\left(\frac{\hbar}{i} \nabla_{1}+\frac{e_{0}}{c} \mathbf{A}(1)\right)^{2}-V_{I}(1)\right] g^{R / A}\left(1,1^{\prime}\right)} \\
& -\int \mathrm{d}^{2} \Sigma^{R / A}(1,2) g^{R / A}\left(2,1^{\prime}\right)=\delta\left(1-1^{\prime}\right),
\end{aligned}
$$

where the functions $\Sigma^{R / A}$ are related to $\Sigma^{\gtrless}$ in analogy to equation $(52) \cdot g^{R / A}$ describe the single-particle spectrum under the influence of the external field, the jellium potential and exchange and correlation effects and are directly related to the spectral function, see definition (48).

\subsection{Gauge-invariant Fourier transform}

It is well known that the electromagnetic field can be introduced in various ways (gauges) which may lead to essentially different explicit forms of the resulting kinetic equations. Although alternative derivations are successfully applied too, gauge invariance becomes a particular problem if the resulting kinetic equations are treated by means of approximations, such as retardation or gradient expansions as in the present case. A critical issue is that the result of these approximations maybe essentially different in different gauges, see e.g. [6] for illustrative examples. To avoid these difficulties, we will formulate the theory in terms of correlation functions which are made explicitly gauge-invariant.

In this section, we use a co-variant 4-vector notation as it makes the following transformations more compact and symmetric. The corresponding definitions are

$$
A_{\mu}=(c \phi, \mathbf{A}), \quad x_{\mu}=(c \tau, \mathbf{r}), \quad X_{\mu}=(c t, \mathbf{R}),
$$

and the conventions $a_{\mu}=\left(a_{0}, \mathbf{a}\right), a^{\mu}=\left(a_{0},-\mathbf{a}\right)$ and $a_{\mu} b^{\mu}=a_{0} b_{0}-\mathbf{a b}$ are being used.

One readily proofs that the Kadanoff-Baym/Keldysh equations (50) remain covariant under gauge transformations, i.e. under the following transformations of the potentials and field operators

$$
A_{\mu}^{\prime}(x)=A_{\mu}(x)-\partial_{\mu} \chi(x), \quad \psi_{a}^{\prime}(x)=\mathrm{e}^{-\frac{i}{\hbar} \frac{e_{0}}{c} \chi(x)} \psi_{a}(x)
$$

From this one immediately obtains the gauge transform of the Green's functions

$$
g^{\prime}(x, X)=\mathrm{e}^{-\frac{\mathrm{i}}{\hbar} \frac{e_{0}}{c}\left[\chi\left(X+\frac{x}{2}\right)-\chi\left(X-\frac{x}{2}\right)\right]} g(x, X) .
$$

Following an idea of Fujita [7], we now introduce a gauge-invariant Green's function $g(\mathbf{k}, X)$ which is given by the modified Fourier transform

$$
\tilde{g}(\mathbf{k}, X)=\int \frac{\mathrm{d}^{4} x}{(2 \pi)^{4}} \exp \left\{\mathrm{i} \int_{-\frac{1}{2}}^{\frac{1}{2}} \mathrm{~d} \lambda x_{\mu}\left[k^{\mu}-\frac{e_{0}}{c} A^{\mu}(X+\lambda x)\right]\right\} g(x, X),
$$


where use has been made of the identity

$$
\chi\left(X+\frac{x}{2}\right)-\chi\left(X-\frac{x}{2}\right)=\int_{-\frac{1}{2}}^{\frac{1}{2}} \mathrm{~d} \lambda \frac{\mathrm{d}}{\mathrm{d} \lambda} \chi(X+\lambda x)=x_{\mu} \partial^{\mu} \int_{-\frac{1}{2}}^{\frac{1}{2}} \mathrm{~d} \lambda \chi(X+\lambda x) .
$$

Indeed, one readily confirms that under any gauge transform (55), the phase factors cancel, and $g^{\prime}(\mathbf{k}, X) \equiv g(\mathbf{k}, X)$.

In the following, we focus on spatially homogeneous electric fields and use the vector potential gauge

$$
A_{0}=\phi=0, \quad \mathbf{A}=-c \int_{-\infty}^{t} \mathrm{~d} \bar{t} \mathbf{E}(\bar{t})
$$

In this case, relation (56) simplifies to

$$
\tilde{g}(\mathbf{k}, \omega ; \mathbf{R}, t)=\int \mathrm{d} \tau \mathrm{d} \mathbf{r} \exp \left[\mathrm{i} \omega \tau-\frac{\mathrm{i}}{\hbar} \mathbf{r} \cdot\left(\mathbf{k}-\frac{e_{0}}{c} \int_{t-\frac{\tau}{2}}^{t+\frac{\tau}{2}} \frac{\mathrm{d} t^{\prime}}{\tau} \mathbf{A}\left(t^{\prime}\right)\right)\right] g(\mathbf{r}, \tau ; \mathbf{R}, t)
$$

what means that the gauge-invariant Green's function $g(\mathbf{k})$ follows from the (conventional) Wigner transformed function $g_{a}(\mathbf{p})$ by replacing the canonical momentum $\mathbf{p}$ by the gauge-invariant kinematic momentum $\mathbf{k}$ according to [9]

$$
\mathbf{p}(t, \tau)=\mathbf{k}-\frac{e_{0}}{c} \int_{t-\frac{\tau}{2}}^{t+\frac{\tau}{2}} \mathrm{~d} t^{\prime} \frac{\mathbf{A}\left(t^{\prime}\right)}{\tau}
$$

Consider two important examples. First, for a constant homogeneous electric field, $\mathbf{E}=\mathbf{E}_{0}$, it follows

$$
\mathbf{A}(t)=-c \mathbf{E}_{0} t, \quad \mathbf{p}(t)=\mathbf{k}+e_{0} \mathbf{E}_{0} t
$$

Second, for a spatially homogeneous harmonic electric field given by $\mathbf{E}(t)=\mathbf{E}_{0} \cos \Omega t$, the vector potential and the momentum relation become, according to equations (57) and (59),

$$
\mathbf{A}(t)=-\frac{c \mathbf{E}_{0}}{\Omega} \sin \Omega t, \quad \mathbf{p}(t, \tau)=\mathbf{k}+\frac{2}{\tau} \frac{e_{0} \mathbf{E}_{0}}{\Omega^{2}} \sin \Omega t \sin \frac{\Omega \tau}{2} .
$$

\subsection{Gauge-invariant quantum kinetic equation for the Wigner distribution}

We are now ready to derive gauge-invariant quantum kinetic equations for the correlation functions $g \gtrless$ and the Wigner distribution. Our starting point is the kinetic equation (50) which we transform to the Wigner representation. For this we assume that macroscopic and microscopic length scales can be separated, where the first is given by the cluster geometry and described by the center of mass variable 
$R$. Gauge-invariant Fourier transformation with respect to the microscopic scale $r$ gives rise to the momentum variable $k$.

In center of mass and relative variables the gradient in the momentum operator in equation (50) becomes $\nabla_{r_{1}}=1 / 2 \nabla_{R}+\nabla_{r}$. Then, applying the spatial part of the transform (58) to the l.h.s. of equation (50) yields

$$
\begin{aligned}
\int \mathrm{d}^{3} r \mathrm{e}^{-\frac{i}{\hbar} \mathbf{r}\left[\mathbf{k}-\frac{e_{0}}{c} \int_{t_{1}^{\prime}}^{t_{1}} \mathrm{~d} \bar{t} \frac{\mathbf{A}(\bar{t})}{t_{1}-t_{1}^{\prime}}\right]}\left[\mathrm{i} \hbar \frac{\partial}{\partial t_{1}}\right. & \left.-\frac{1}{2 m_{\mathrm{e}}}\left(\frac{\hbar}{\mathrm{i}} \nabla_{r}+\frac{1}{2} \frac{\hbar}{\mathrm{i}} \nabla_{R}+\frac{e_{0}}{c} \mathbf{A}(1)\right)^{2}-V_{I}(1)\right] \\
& \times \int \frac{\mathrm{d}^{3} k_{1}}{(2 \pi \hbar)^{3}} \mathrm{e}^{\frac{\mathrm{i}}{\hbar} \mathbf{r}\left[\mathbf{k}_{1}-\frac{e_{0}}{c} \int_{t_{1}^{\prime}}^{t_{1}} \mathrm{~d} \overline{\frac{\mathbf{A}(\bar{t})}{t_{1}-t_{1}^{\prime}}}\right]} \tilde{g} \gtrless\left(\mathbf{R}, \mathbf{k}_{1}, t_{1}, t_{1}^{\prime}\right) .
\end{aligned}
$$

The $t_{1}$ and $r$ derivatives on the second exponential are readily performed, and we notice that the vector potential terms in the exponentials cancel. Further, using $\mathbf{r}=\hbar / \mathrm{i} \nabla_{\mathbf{k}_{1}}$, the $r$-integration can be performed, except for the potential term, with the result (details of the derivation are given in the appendix)

$$
\begin{gathered}
\left\{\left[\mathrm{i} \hbar \frac{\partial}{\partial t_{1}}+\mathrm{i} \hbar \frac{\mathbf{K}^{A}\left(t_{1}, t_{1}^{\prime}\right)}{t_{1}-t_{1}^{\prime}} \nabla_{\mathbf{k}}\right]-\frac{1}{2 m_{\mathrm{e}}}\left[\frac{1}{2} \frac{\hbar}{\mathrm{i}} \nabla_{R}+\mathbf{k}+\mathbf{K}^{A}\left(t_{1}, t_{1}^{\prime}\right)\right]^{2}\right\} \tilde{g}^{\gtrless}\left(\mathbf{R}, \mathbf{k}, t_{1}, t_{1}^{\prime}\right) \\
-\int \mathrm{d}^{3} r \int \frac{\mathrm{d}^{3} k_{1}}{(2 \pi \hbar)^{3}} \mathrm{e}^{-\frac{\mathrm{i}}{\hbar} \mathbf{r}\left(\mathbf{k}-\mathbf{k}_{1}\right)} V_{I}^{\mathrm{eff}}\left(\mathbf{R}+\frac{\mathbf{r}}{2}, t_{1}\right) \tilde{g}^{\gtrless}\left(\mathbf{R}, \mathbf{k}_{1}, t_{1}, t_{1}^{\prime}\right)= \\
=\tilde{I} \gtrless\left(\mathbf{R}, \mathbf{k}, t_{1}, t_{1}^{\prime}\right)+\tilde{I}_{\mathrm{F}}^{\gtrless}\left(\mathbf{R}, \mathbf{k}, t_{1}, t_{1}^{\prime}\right),
\end{gathered}
$$

where the field-dependent momentum $K^{A}$ is defined by

$$
\mathbf{K}^{A}\left(t, t^{\prime}\right) \equiv-\frac{e_{0}}{c} \int_{t^{\prime}}^{t} \mathrm{~d} t^{\prime \prime} \frac{\mathbf{A}(t)-\mathbf{A}\left(t^{\prime \prime}\right)}{t-t^{\prime}}
$$

For the above two examples of a constant and monochromatic field, equations $(60,61)$, respectively, this has the form

$$
\begin{aligned}
& \mathbf{K}^{A}\left(t, t^{\prime}\right)=e_{0} \mathbf{E}_{0} \frac{t-t^{\prime}}{2}, \quad \mathbf{E} \equiv \mathbf{E}_{0} \\
& \mathbf{K}^{A}\left(t, t^{\prime}\right)=\frac{e_{0} \mathbf{E}_{0}}{\Omega^{2}\left(t-t^{\prime}\right)}\left\{\Omega\left(t-t^{\prime}\right) \sin \Omega t+\cos \Omega t-\cos \Omega t^{\prime}\right\}, \quad \mathbf{E} \equiv \mathbf{E}_{0} \cos \Omega t .
\end{aligned}
$$

In equation (63) we introduced an effective time-dependent confinement potential which includes the Hartree mean field,

$$
\begin{aligned}
V_{I}^{\mathrm{eff}}(\mathbf{R}, t) & \equiv V_{I}(\mathbf{R}, t)+\Sigma^{\mathrm{H}}(\mathbf{R}, t), \\
\Sigma^{\mathrm{H}}(\mathbf{R}, t) & =\int \mathrm{d} \mathbf{r} V(\mathbf{R}-\mathbf{r}) n(\mathbf{r}, t)=\int \mathrm{d}^{3} r \frac{\mathrm{d}^{3} k}{(2 \pi \hbar)^{3}} V(\mathbf{R}-\mathbf{r}) f(\mathbf{r}, \mathbf{k}, t),
\end{aligned}
$$

which depends on the nonequilibrium electron density profile. Finally, the r.h.s. of equation (63) contains all remaining two-particle and higher order contributions 
to the dynamics (i.e. collisions and exchange mean field) the explicit structure of which will be discussed below in section 3.7. These gauge-invariant equations of motion for the two-time correlation functions are the basis for all further analysis. In principle, their direct numerical solution is possible, following previous numerical work for spatially homogeneous charged particle systems, e.g. [2,22]. Here, however, we concentrate on the derivation of a closed equation of motion for the (single-time) Wigner function as it is essentially more simple.

To obtain from equation (63) the gauge-invariant equation for the single-time distribution function we also need the adjoint equation and compute the difference of the two, e.g. [2,3]. Taking this difference equation for $g^{<}$at equal times, $t_{1}=t_{1}^{\prime}$, allows us to express $g^{<}$by the Wigner distribution according to relation (47) with the result (details are given in the appendix)

$$
\begin{aligned}
\left\{\frac{\partial}{\partial t}+\right. & \left.\frac{\mathbf{k}}{m_{\mathrm{e}}} \nabla_{R}-e_{0} \mathbf{E}(t) \nabla_{\mathbf{k}}\right\} f(\mathbf{R}, \mathbf{k}, t) \\
& +\frac{2}{\hbar} \int \mathrm{d}^{3} r \int \frac{\mathrm{d}^{3} k_{1}}{(2 \pi \hbar)^{3}} \sin \frac{\mathbf{r}\left(\mathbf{k}-\mathbf{k}_{1}\right)}{\hbar} V_{I}^{\mathrm{eff}}\left(\mathbf{R}+\frac{\mathbf{r}}{2}, t\right) f\left(\mathbf{R}, \mathbf{k}_{1}, t\right)= \\
& =I(\mathbf{R}, \mathbf{k}, t)+I_{\mathrm{F}}(\mathbf{R}, \mathbf{k}, t),
\end{aligned}
$$

where $I(\mathbf{R}, \mathbf{k}, t) \equiv-2 \operatorname{Re}\left[\tilde{I}^{<}(\mathbf{R}, \mathbf{k}, t, t)\right]$ and $I_{\mathrm{F}}(\mathbf{R}, \mathbf{k}, t) \equiv-2 \operatorname{Re}\left[\tilde{I}_{\mathrm{F}}^{<}(\mathbf{R}, \mathbf{k}, t, t)\right]$. Here $E$ is the total electric field (external plus induced) which obeys Maxwell's equations which have to be solved selfconsistently with the kinetic equation (69).

Equation (69) is not yet the final equation for the Wigner distribution which will be presented below, in section 3.8, after evaluation of the collision and exchange terms in section 3.7. But before doing this, we analyze the term with the external potential (the integral on the l.h.s.) more in detail. The integral representation of $V_{I}^{\text {eff }}$ in equation (69) is still completely general and does not use any assumptions on the space dependence of the confinement potential. The present form applies even to sharp spatial changes and can be used with any form of pseudo-potentials for the core ions of clusters and it fully includes quantum effects.

Analytical simplifications are possible in the case of the jellium potential due to its smooth variation in space. In that case it is possible to eliminate the integrations over the jellium potential by expanding $V_{I}^{\text {eff }}$ around the center of mass coordinate, e.g. [2],

$$
V_{I}^{\mathrm{eff}}\left(\mathbf{R} \pm \frac{\mathbf{r}}{2}\right)=\sum_{l=0}^{\infty} \frac{( \pm 1)^{l}}{2^{l} l !} \frac{\partial^{l} V_{I}^{\mathrm{eff}}(\mathbf{R})}{\partial \mathbf{R}_{i} \partial \mathbf{R}_{j} \ldots \partial \mathbf{R}_{l}} \cdot \mathbf{r}_{i} \mathbf{r}_{j} \ldots \mathbf{r}_{l}
$$

where $\mathbf{r}_{j} \partial V_{I}^{\text {eff }} / \partial \mathbf{R}_{j}$ denotes the scalar product $\nabla_{\mathbf{R}_{j}} V_{I}^{\text {eff }} \cdot \mathbf{r}_{j}$ and so on. With this, the integral term in equation (69) becomes

$$
\begin{array}{r}
\frac{2}{\hbar} \int \mathrm{d}^{3} r \int \frac{\mathrm{d}^{3} k_{1}}{(2 \pi \hbar)^{3}} \sin \frac{\mathbf{r}\left(\mathbf{k}-\mathbf{k}_{1}\right)}{\hbar} V_{I}^{\mathrm{eff}}\left(\mathbf{R}+\frac{\mathbf{r}}{2}, t\right) f\left(\mathbf{R}, \mathbf{k}_{1}, t\right)= \\
=-\sum_{l=0}^{\infty} \frac{(\mathrm{i} \hbar)^{2 l+1}}{2^{2 l}(2 l+1) !} \frac{\partial^{2 l+1} V_{I}^{\mathrm{eff}}(\mathbf{R}, t)}{\partial \mathbf{R}_{i} \partial \mathbf{R}_{j} \ldots \partial \mathbf{R}_{2 l+1}} \cdot \frac{\partial^{2 l+1} f(\mathbf{R}, \mathbf{k}, t)}{\partial k_{i} \partial k_{j} \ldots \partial k_{2 l+1}}
\end{array}
$$




$$
=-\left\{\nabla_{\mathbf{R}} V_{I}^{\mathrm{eff}}(\mathbf{R}, t) \nabla_{\mathbf{k}}-\frac{\hbar^{2}}{24} \frac{\partial^{3} V_{I}^{\mathrm{eff}}(\mathbf{R}, t)}{\partial \mathbf{R}_{1} \partial \mathbf{R}_{2} \partial \mathbf{R}_{3}} \cdot \frac{\partial^{3}}{\partial \mathbf{k}_{1} \partial \mathbf{k}_{2} \partial \mathbf{k}_{3}} \ldots\right\} f(\mathbf{R}, \mathbf{k}, t) .
$$

Applying this expansion to the jellium potential (10) we readily see that the first term of the expansion of $V_{I}^{\text {eff }}$ is dominating: indeed, inside the cluster where $V_{I}$ is parabolic, the third derivative vanishes exactly. Outside, where $V_{I}$ is Coulomb-like, the third derivative is short range, it decays as $1 / r^{4}$ and gives only a small correction. We may expect that more realistic pseudopotentials will behave similarly since they do not exhibit sharp changes or even divergencies [13].

\subsection{Gauge-invariant expression for the Hartree-Fock and collision terms. Nonlocal corrections}

We now perform the gauge-invariant Fourier transform of the right hand side of the kinetic equation which contains all two-particle quantities related to mean field and correlation effects. Let us start with the collision integral. If the characteristic length scales of the scattering processes are small compared to the cluster radius, the local approximation can be used. Then the collision integrals retain their form of the homogeneous case with $R$ being an additional parameter, and our previous results $[8,9]$ can be applied leading to

$$
\begin{aligned}
I(\mathbf{R}, \mathbf{k}, t)= & -2 \operatorname{Re} \int_{t_{0}}^{t} \mathrm{~d} \bar{t}\left\{\Sigma^{>}\left[\mathbf{R}, \mathbf{k}+\mathbf{K}^{A}(t, \bar{t}), t, \bar{t}\right] g^{<}\left[\mathbf{R}, \mathbf{k}+\mathbf{K}^{A}(t, \bar{t}), \bar{t}, t\right]\right. \\
& \left.-\Sigma^{<}\left[\mathbf{R}, \mathbf{k}+\mathbf{K}^{A}(t, \bar{t}), t, \bar{t}\right] g^{>}\left[\mathbf{R}, \mathbf{k}+\mathbf{K}^{A}(t, \bar{t}), \bar{t}, t\right]\right\} .
\end{aligned}
$$

Note that the momentum arguments in all functions are shifted by the field-dependent momentum $K^{A}$ which reflects the explicit field-dependence of the two-particle scattering process. This is an important effect leading to the so-called intracollisional field effect and to nonlinear phenomena including collisional harmonics generation, (inverse) bremsstrahlung, multiphoton excitation and ionization etc $[8,9]$.

The result (72) gives the local approximation for the collision integral. It is however, straightforward to write down the first gradient corrections. For this we recognize that (72) is of the general structure (45), except for the time integral. We thus can write [using the notation of equation (45)]

$$
\begin{aligned}
I_{\text {grad }}(\mathbf{R}, \mathbf{k}, t)= & \hbar \operatorname{Im} \int_{t_{0}}^{t} \mathrm{~d} \bar{t}\left(\nabla_{\mathbf{R}_{1}} \nabla_{\mathbf{k}_{2}}-\nabla_{\mathbf{R}_{2}} \nabla_{\mathbf{k}_{1}}\right) \\
& \times\left\{\Sigma^{>}\left[\mathbf{R}_{1}, \mathbf{k}_{1}+\mathbf{K}^{A}(t, \bar{t}), t, \bar{t}\right] g^{<}\left[\mathbf{R}_{2}, \mathbf{k}_{2}+\mathbf{K}^{A}(t, \bar{t}), \bar{t}, t\right]\right. \\
& \left.-\Sigma^{<}\left[\mathbf{R}_{1}, \mathbf{k}_{1}+\mathbf{K}^{A}(t, \bar{t}), t, \bar{t}\right] g^{>}\left[\mathbf{R}_{2}, \mathbf{k}_{2}+\mathbf{K}^{A}(t, \bar{t}), \bar{t}, t\right]\right\}\left.\right|_{(\mathbf{R}, \mathbf{k})} .
\end{aligned}
$$

This result has to be added to the local approximation (72).

Expressions $(72,73)$ are still completely general. A particular collision process is specified by the appropriate choice of the selfenergies. Further, the two-time correlation functions have to be expressed by the Wigner distributions via the so-called reconstruction ansatz. Its original form due to Baym and Kadanoff [3] was generalized 
by Lipavski, Spicka and Velicky [33] to nonequilibrium systems, properly accounting for causality and retardation effects. The latter ansatz was further generalized to the presence of external fields [6,9], and here we account, in addition, for weak spatial inhomogeneity which gives rise to gradient corrections in the reconstruction ansatz:

$$
\begin{aligned}
\pm g^{\gtrless}\left(\mathbf{R}, \mathbf{k}, t, t^{\prime}\right)= & g^{R}\left(\mathbf{R}, \mathbf{k}, t, t^{\prime}\right) f^{\gtrless}\left[\mathbf{R}, \mathbf{k}-\mathbf{K}^{A}\left(t^{\prime}, t\right), t^{\prime}\right] \\
& -f \gtrless\left[\mathbf{R}, \mathbf{k}-\mathbf{K}^{A}\left(t, t^{\prime}\right), t\right] g^{A}\left(\mathbf{R}, \mathbf{k}, t, t^{\prime}\right)+\frac{\mathrm{i} \hbar}{2}\left(\nabla_{\mathbf{R}_{1}} \nabla_{\mathbf{k}_{2}}-\nabla_{\mathbf{R}_{2}} \nabla_{\mathbf{k}_{1}}\right) \\
& \times\left\{g^{R}\left(\mathbf{R}_{1}, \mathbf{k}_{1}, t, t^{\prime}\right) f^{\gtrless}\left[\mathbf{R}_{2}, \mathbf{k}_{2}-\mathbf{K}^{A}\left(t^{\prime}, t\right), t^{\prime}\right]\right. \\
& \left.-f \gtrless\left[\mathbf{R}_{1}, \mathbf{k}_{1}-\mathbf{K}^{A}\left(t, t^{\prime}\right), t\right] g^{A}\left(\mathbf{R}_{2}, \mathbf{k}_{2}, t, t^{\prime}\right)\right\}\left.\right|_{(\mathbf{R}, \mathbf{k})},
\end{aligned}
$$

where $f^{>} \equiv 1-f$ and $f^{<} \equiv f$.

Consider now the Hartree-Fock terms. They have a product form analogous to the collision term. Furthermore, due to the time-diagonal structure of $\Sigma^{\mathrm{HF}}$, the fieldinduced momentum shift $K^{A}$ vanishes $\left(K^{A}(t, t)=0\right)$. In particular, the Hartree term can be written as (see appendix)

$$
\Sigma^{\mathrm{H}}\left(\mathbf{r}_{1}, t_{1} ; \mathbf{r}_{1}^{\prime}, t_{1}^{\prime}\right)=\delta\left(\mathbf{r}_{1}-\mathbf{r}_{1}^{\prime}\right) \delta\left(t_{1}-t_{1}^{\prime}\right) \int \mathrm{d}^{3} r_{2} V\left(\mathbf{r}_{1}-\mathbf{r}_{2}\right) n\left(r_{2}, t\right) .
$$

This potential is local in space and is treated exactly like the jellium potential $V_{I}$. Both terms can be combined into an effective confinement potential $V_{I}^{\text {eff }}$, see equation (82) below.

Let us now turn to the nonequilibrium exchange term which is given by

$$
\begin{aligned}
\Sigma^{\mathrm{F}}\left(\mathbf{r}_{1}, t_{1} ; \mathbf{r}_{1}^{\prime}, t_{1}^{\prime}\right) & =\delta\left(t_{1}-t_{1}^{\prime}\right) \Sigma^{\mathrm{F}}\left(\mathbf{r}_{1}, \mathbf{r}_{1}^{\prime}, t_{1}\right), \\
\Sigma^{\mathrm{F}}\left(\mathbf{r}_{1}, \mathbf{r}_{1}^{\prime}, t\right) & =-V\left(\mathbf{r}_{1}-\mathbf{r}_{1}^{\prime}\right) f\left(\mathbf{r}_{1}, \mathbf{r}_{1}^{\prime}, t\right),
\end{aligned}
$$

and has the Fourier representation

$$
\Sigma^{\mathrm{F}}(\mathbf{R}, \mathbf{k}, t)=-\int \frac{\mathrm{d}^{3} k_{2}}{(2 \pi \hbar)^{3}} V\left(k_{2}\right) f\left(\mathbf{R}, \mathbf{k}-\mathbf{k}_{2}, t\right) .
$$

In the kinetic equation it appears in the combination

$$
I^{\mathrm{F}}(\mathbf{R}, \mathbf{k}, t)=-2 \operatorname{Re}\left[\Sigma^{\mathrm{F}}(\mathbf{R}, \mathbf{k}, t) g^{<}(\mathbf{R}, \mathbf{k}, t, t)\right],
$$

which vanishes exactly (on the time-diagonal, $g^{<}$is purely imaginary). This means that the only nonzero exchange contributions arise from the gradient corrections which appear due to the product $\Sigma^{\mathrm{F}} \cdot g^{<}$. Using the general expression (45), we obtain

$$
\begin{aligned}
I^{\mathrm{F}}(\mathbf{R}, \mathbf{k}, t)= & \left.2 \operatorname{Re}\left(1+\frac{\mathrm{i} \hbar}{2}\{\ldots\}\right) \Sigma^{F}\left(\mathbf{R}_{1}, \mathbf{Q}_{1}, t\right) g^{<}\left(\mathbf{R}_{2}, \mathbf{Q}_{2}, t, t\right)\right|_{(\mathbf{R}, \mathbf{k})}= \\
= & \int \frac{\mathrm{d}^{3} k_{1}}{(2 \pi \hbar)^{3}} V\left(k_{1}\right)\left\{\nabla_{\mathbf{R}_{1}} f\left(\mathbf{R}_{1}, \mathbf{Q}_{1}-\mathbf{k}_{1}, t\right) \nabla_{\mathbf{Q}_{2}} f\left(\mathbf{R}_{2}, \mathbf{Q}_{2}, t\right)\right. \\
& \left.-\nabla_{\mathbf{Q}_{1}} f\left(\mathbf{R}_{1}, \mathbf{Q}_{1}-\mathbf{k}_{1}, t\right) \nabla_{\mathbf{R}_{2}} f\left(\mathbf{R}_{2}, \mathbf{Q}_{2}, t\right)\right\}\left.\right|_{(\mathbf{R}, \mathbf{k})} .
\end{aligned}
$$


Of the two terms in curly brackets, the first has the form of the gradient of a local exchange potential, $\nabla_{\mathbf{R}} \Sigma^{F}(\mathbf{R}, \mathbf{k}) \cdot \nabla_{\mathbf{k}} f(\mathbf{R}, \mathbf{k})$. This term may be included into the gradient of a local total potential together with the confinement and Hartree field, $V_{I}^{\text {tot }}(\mathbf{R}, \mathbf{k}, t)=V_{I}(\mathbf{R})+\Sigma^{\mathrm{H}}(\mathbf{R}, t)+\Sigma^{F}(\mathbf{R}, \mathbf{k}, t)$. However, the second term appears to be of the same order and cannot be neglected if the first is retained ${ }^{11}$. This second contribution has the form $\nabla_{\mathbf{k}} \Sigma^{F}(\mathbf{R}, \mathbf{k}) \cdot \nabla_{\mathbf{R}} f(\mathbf{R}, \mathbf{k})$ and effectively renormalizes the velocity in the drift term of the kinetic equation, $\mathbf{k} / m_{\mathrm{e}} \nabla_{\mathbf{R}} f \rightarrow$ $\left[\mathbf{k} / m_{\mathrm{e}}+\nabla_{\mathbf{k}} \Sigma^{F}(\mathbf{R}, \mathbf{k})\right] \nabla_{\mathbf{R}} f$. We may further generalize the drift term by permitting a general single-particle energy dispersion (or band structure) $k^{2} / 2 m_{\mathrm{e}} \rightarrow \epsilon(\mathbf{k})$, as our derivation did not depend on the explicit form. Finally, we can write the drift term as $\nabla_{\mathbf{k}} \bar{\epsilon}(\mathbf{k})$ with the effective single-particle energy being renormalized by the Hartree-Fock and confinement energy (using the fact that $\Sigma^{\mathrm{H}}$ and $V_{I}$ are momentumindependent $), \bar{\epsilon}(\mathbf{R}, \mathbf{k}, t) \equiv \epsilon(\mathbf{k})+\Sigma^{\mathrm{H}}(\mathbf{R}, t)+\Sigma^{F}(\mathbf{R}, \mathbf{k}, t)+V_{I}(\mathbf{R})$. Finally, the gradient of the effective potential can be written in terms of the same effective single-particle energy, since the kinetic energy is R-independent, $\nabla_{\mathbf{R}} V_{I}^{\text {eff }}=\nabla_{\mathbf{R}} \bar{\epsilon}$, allowing for a highly symmetric form of the kinetic equation ${ }^{12}$.

\subsection{Resulting quantum kinetic equation}

Collecting all terms together, the resulting kinetic equation which includes terms up to first order gradients reads

$$
\begin{aligned}
& \left\{\frac{\partial}{\partial t}+\nabla_{\mathbf{k}} \bar{\epsilon}(\mathbf{k}, \mathbf{R}, t) \nabla_{R}-\left[e_{0} \mathbf{E}(t)+\nabla_{\mathbf{R}} \bar{\epsilon}(\mathbf{R}, \mathbf{k}, t)\right] \nabla_{\mathbf{k}}\right\} f(\mathbf{R}, \mathbf{k}, t)=\bar{I}(\mathbf{R}, \mathbf{k}, t), \\
& \bar{\epsilon}(\mathbf{R}, \mathbf{k}, t) \equiv \epsilon(\mathbf{k})+V_{I}^{\mathrm{eff}}(\mathbf{R}, t)+\Sigma^{\mathrm{F}}(\mathbf{R}, \mathbf{k}, t), \\
& V_{I}^{\mathrm{eff}}(\mathbf{R}, t) \equiv V_{I}(R)+\Sigma^{\mathrm{H}}(\mathbf{R}, t), \\
& \bar{I}(\mathbf{R}, \mathbf{k}, t) \equiv I(\mathbf{R}, \mathbf{k}, t)+I_{\mathrm{grad}}(\mathbf{R}, \mathbf{k}, t),
\end{aligned}
$$

where $\Sigma^{\mathrm{H}}, \Sigma^{F}, I$ and $I_{\text {grad }}$ have been defined in equations (72), (73), (75), (77).

We underline that this equation is very general. It applies to arbitrary nonequilibrium situations and strong electromagnetic fields with arbitrary amplitude and time-dependence (the only assumption is that the field does not vary significantly on the space scale $R_{I}$ of the cluster). Due to the gauge-invariant derivation, the resulting kinetic equation with all gradient correcations is gauge-invariant as well. This equation is internally consistent on the level of first gradients. The neglect of higher order spatial derivatives assumes that $R_{I}\left|\nabla^{2} n(R)\right| \ll|\nabla n(R)|$.

Equation (80) directly generalizes the density functional results considered above to nonequilibrium situations and the presence of a strong field: in particular, the total energy $E_{\mathrm{ve}}[n(r)]$ appearing in equation (14) is generalized to the nonequilibrium

\footnotetext{
${ }^{11}$ In particular, the use - in a classical Vlasov equation - of an effective potential which includes exchange, as done by several authors, e.g. [27,28], while neglecting the second gradient contribution in equation (79) can not be justified from the perspective of quantum kinetic theory.

${ }^{12}$ The general structure is, of course, the same as in the quasiparticle equations of Landau Fermi liquid theory.
} 
expression $\bar{\epsilon}[f]$ depending on the nonequilibrium momentum-dependent Wigner distribution instead of the local density:

$$
E(\mathbf{R}, t)=\int \frac{\mathrm{d}^{3} k}{(2 \pi \hbar)^{3}} \bar{\epsilon}(\mathbf{R}, t,[f(\mathbf{R}, \mathbf{k}, t)]) f(\mathbf{R}, \mathbf{k}, t)+U_{\text {cor }}(\mathbf{R}, t),
$$

where the correlation energy contribution $U_{\text {cor }}$ arises from the collision integral.

Finally, we discuss possible generalizations of the above kinetic equation. The main simplification involved in the derivation of equation (80) is the restriction to first order gradient corrections. On the other hand, we can use the full quantum result for the local potential and the Hartree term. The exchange contribution, however, cannot be included since it is momentum dependent and has a gradient expansion completely different from that of $V_{I}^{\text {eff }}$

$$
\begin{aligned}
& \left\{\frac{\partial}{\partial t}+\nabla_{\mathbf{k}} \epsilon(\mathbf{k}) \nabla_{R}-e_{0} \mathbf{E}(t) \nabla_{\mathbf{k}}\right\} f(\mathbf{R}, \mathbf{k}, t)+\hat{I}^{F}\left(\left[\Sigma^{F}\right],[f]\right) \\
& \quad+\frac{2}{\hbar} \int \mathrm{d}^{3} r \int \frac{\mathrm{d}^{3} k_{1}}{(2 \pi \hbar)^{3}} \sin \frac{\mathbf{r}\left(\mathbf{k}-\mathbf{k}_{1}\right)}{\hbar} V_{I}^{\mathrm{eff}}\left(\mathbf{R}+\frac{\mathbf{r}}{2}, t\right) f\left(\mathbf{R}, \mathbf{k}_{1}, t\right)=\hat{I}(\mathbf{R}, \mathbf{k}, t), \\
& V_{I}^{\mathrm{eff}}(\mathbf{R}, \mathbf{k}, t) \equiv V_{I}(R)+\Sigma^{\mathrm{H}}(\mathbf{R}, t) .
\end{aligned}
$$

Here the integral term with $V_{I}^{\text {eff }}$ contains gradient corrections of all orders, the first two were given in equation (71), and $\hat{I}^{F}$ and $\hat{I}$ denote the gradient expansions of the exchange term and the collision integral, respectively, the first orders of which were given above by equations (73), (79). To go beyond the kinetic equation (80) in a strict manner would require to retain in all terms all contributions up to a given derivative $\partial^{k} f / \partial R^{k}$ which can be expected to be total energy and sum rule conserving and fully internally consistent. On the other hand, simpler schemes seem possible. First of all a simplified treatment of the collision integral compared to the remaining terms, (e.g. by retaining only the 0th or 0th plus 1st gradient term) seems reasonable for most applications. Secondly, a treatment of the Fock term on the level of first gradients (cf. equation (80) should capture the dominant exchange effects. At the same time, a full quantum treatment of $V_{I}^{\text {eff }}$ is possible in order to analyze the role of quantum effects such as tunneling etc.

\subsection{Spectral properties of the valence electrons. Propagator in a strong field}

The strength of the Green's functions approach is that statistical properties - given by the Wigner distribution - and dynamical information - related to the spectral function or density of states - are treated fully selfconsistently. This is of particular importance if approximations are being developed. How the spectral information enters the Green's functions is seen explicitly in the reconstruction relation (74), [although it is an approximation]. Here the spectral information is contained in the retarded and advanced Green's functions which, consequently, have to be determined together with the distribution function. 
Indeed, both the equations of motion for $f$, equation (80), and $g^{R / A}$ are derived from the more general equations of motion for the two-time correlations functions $g^{\gtrless}\left(t, t^{\prime}\right)$, the Kadanoff-Baym/Keldysh equation (63) and its adjoint, equation (131). So far we considered only one possible combination of the two - its difference, which resulted in the equation of motion for the Wigner distribution (taking the difference, yields essentially an equation containing a commutator of $f$ and the hamiltonian). Also, in taking this equation only on the time diagonal, $t=t^{\prime}$ or, equivalently, $\tau=0$ by no means exhausted the full information contained in the two-time funtions.

\subsubsection{Gauge-invariant equation of motion for the retarded Green's function}

The additional spectral information is recovered by considering the Green's functions away from the time diagonal, i.e. as a function of the relative time $\tau$. This is most easily analyzed by computing the sum of equation (63) and its adjoint. The result is (details are given in the appendix)

$$
\begin{aligned}
\{\mathrm{i} \hbar & \frac{\partial}{\partial \tau}+\mathrm{i} \hbar \frac{\mathbf{K}_{+}^{A}\left(t_{1}, t_{1}^{\prime}\right)}{t_{1}-t_{1}^{\prime}} \nabla_{\mathbf{k}}-\frac{1}{2 m_{\mathrm{e}}}\left[\left(\frac{\hbar}{2 \mathrm{i}} \nabla_{\mathbf{R}}\right)^{2}+k^{2}+\frac{\left(\mathbf{K}^{A}\right)^{2}\left(t_{1}, t_{1}^{\prime}\right)+\left(\mathbf{K}^{A}\right)^{2}\left(t_{1}^{\prime}, t_{1}\right)}{2}\right. \\
& \left.\left.+\frac{\hbar}{\mathrm{i}} \mathbf{K}_{-}^{A}\left(t_{1}, t_{1}^{\prime}\right) \nabla_{\mathbf{R}}+2 \mathbf{K}_{+}^{A}\left(t_{1}, t_{1}^{\prime}\right) \mathbf{k}\right]\right\} \tilde{g}^{R / A}\left(\mathbf{R}, \mathbf{k}, t_{1}, t_{1}^{\prime}\right) \\
& -\frac{1}{2} \int \mathrm{d}^{3} r \int \frac{\mathrm{d}^{3} k_{1}}{(2 \pi \hbar)^{3}}\left\{\mathrm{e}^{-\mathrm{i} \frac{\mathbf{r}\left(\mathbf{k}-\mathbf{k}_{1}\right)}{\hbar}} V_{I}^{\mathrm{eff}}\left(\mathbf{R}+\frac{\mathbf{r}}{2}, t_{1}\right)+\mathrm{e}^{\mathrm{i} \frac{\mathbf{r}\left(\mathbf{k}-\mathbf{k}_{1}\right)}{\hbar}} V_{I}^{\text {eff }}\left(\mathbf{R}-\frac{\mathbf{r}}{2}, t_{1}^{\prime}\right)\right\} \\
& \times \tilde{g}^{R / A}\left(\mathbf{R}, \mathbf{k}_{1}, t_{1}, t_{1}^{\prime}\right)=I_{-}^{R / A}\left(\mathbf{R}, \mathbf{k}, t_{1}, t_{1}^{\prime}\right)+\delta\left(t_{1}-t_{1}^{\prime}\right),
\end{aligned}
$$

where the definition of $I_{-}^{R / A}$ is analogous to that of $I_{-}^{\gtrless}$ and we introduced

$$
\mathbf{K}_{ \pm}^{A}\left(t, t^{\prime}\right) \equiv\left[\mathbf{K}^{A}\left(t, t^{\prime}\right) \pm \mathbf{K}^{A}\left(t^{\prime}, t\right)\right] .
$$

From the retarded and advanced Green's functions (in fact, due to the symmetry (53) knowledge of one function in one half-plane, e.g. knowledge of $g^{R}$ for $\tau \geqslant 0$ is sufficient) the complete spectral information is known. In particular, the spectral function follows from the definition (48). The result (87) is still completely general. It includes strong field effects, spatial inhomogeneities and correlations. Analytical expressions are of special interest, and we now consider some important cases.

\subsubsection{Spectral function for a homogeneous system in an external field}

Consider first the simplest case of a spatially homogenous ideal electron gas (no mean field and collisions) in the absence of confinement and external fields, i.e. $K^{A}=\nabla_{R}=V_{I}=0$. The resulting equation is readily solved

$$
\left\{\mathrm{i} \hbar \frac{\partial}{\partial \tau}-\frac{k^{2}}{2 m_{\mathrm{e}}}\right\} g^{R}(k, \tau, t)=\delta(\tau), \quad g^{R}(k, \tau)=-\frac{\mathrm{i}}{\hbar} \Theta(\tau) \mathrm{e}^{-\frac{\mathrm{i}}{\hbar} \frac{k^{2}}{2 m_{\mathrm{e}}} \tau} .
$$

With the definition (48) this yields the spectral function

$$
a(k, \tau)=\mathrm{e}^{-\frac{\mathrm{i}}{\hbar} \frac{k^{2}}{2 m_{\mathrm{e}}} \tau},
$$


corresponding, in frequency space, to a sharp energy spectrum,

$a(k, \omega)=2 \pi \hbar \delta\left(\hbar \omega-k^{2} /\left(2 m_{\mathrm{e}}\right)\right)$, which is peaked at the free single-particle energy. Next, we include mean field effects (and possible band structure effects) which does not change the structure, except for a possible slow (macroscopic) time-dependence of the effective single-particle energy (which includes the Hartree-Fock energy) via the Wigner distribution,

$$
\left\{\mathrm{i} \hbar \frac{\partial}{\partial \tau}-\epsilon(\mathbf{k}, t)\right\} g^{R}(\mathbf{k}, \tau, t)=\delta(\tau), \quad a(\mathbf{k}, \tau, t)=\mathrm{e}^{-\frac{\mathrm{i}}{\hbar} \int_{t-\tau / 2}^{t+\tau / 2} \mathrm{~d} \bar{\epsilon} \epsilon(\mathbf{k}, \bar{t})} .
$$

The corresponding frequency spectrum again consists of a single sharp line, now shifted from $k^{2} / 2 m$ to $\epsilon(\mathbf{k}, t)$.

Further, correlation effects (related to the collision integral) cause a damping of the spectral function (91), so the oscillations in $\tau$ are modulated with an overall decay with increasing $|\tau|$ which is naturally interpreted as a finite life time of the (quasi-)particle. Correspondingly, in frequency space, a broadening of the peak is observed. A detailed analysis has been given, e.g. in [2,31]. Here we only recall the main result: an intuitive inclusion of damping by a constant exponential factor $\mathrm{e}^{-\gamma|\tau|}$ leading to a replacement of the energy delta function by a Lorentzian, gives rise to unphysical results which are due to the slow decay at large frequencies of that spectral function. Therefore, improved analytical results have been derived [31] which have a zero slope at the time diagonal $\tau=0$ and cure this defect.

For our present analysis, where we are interested in the effect of intense electromagnetic fields on the spectrum, we expect that a detailed selfconsistent treatment of the correlation effects on the spectral function can be avoided. As was found in many investigations, in strong fields inclusion of all external fields into the spectral function together with Hartree-Fock effects, is crucial for a correct modeling of the many-particle behavior, see e.g. [32]. Correlation effects lead to an energy shift and broadening which is generally well understood. Therefore, these effects can be added to the collisionless result in perturbation theory. We will thus concentrate in the following on the first step of perturbation theory where collision effects on the spectral function are neglected.

The next step is to include, in addition to the single-particle energy, an external spatially homogeneous field. Then, the equation reads

$$
\begin{aligned}
& \left\{\mathrm{i} \hbar \frac{\partial}{\partial \tau}+\frac{\mathrm{i} \hbar}{\tau} \mathbf{K}_{+}^{A}\left(t_{1}, t_{1}^{\prime}\right) \nabla_{\mathbf{k}}\right. \\
& \left.\quad-\frac{1}{4 m_{\mathrm{e}}}\left[\left(\mathbf{k}+\mathbf{K}^{A}\left(t_{1}, t_{1}^{\prime}\right)\right)^{2}+\left(\mathbf{k}+\mathbf{K}^{A}\left(t_{1}^{\prime}, t_{1}\right)\right)^{2}\right]\right\} g^{R}\left(\mathbf{R}, \mathbf{k}, t_{1}, t_{1}^{\prime}\right)=\delta(\tau),
\end{aligned}
$$

and again a solution is possible, e.g. [9],

$$
a(k, \tau, t)=\exp \left\{-\frac{\mathrm{i}}{4 m_{\mathrm{e}} \hbar} \int_{t-\tau / 2}^{t+\tau / 2} \mathrm{~d} \bar{t}\left[2 k^{2}+\left(\mathbf{K}^{A}\left(\bar{t}, t^{\prime}\right)\right)^{2}+\left(\mathbf{K}^{A}\left(t^{\prime}, \bar{t}\right)\right)^{2}\right]\right\}
$$


One readily obtains the results for limiting cases: for a constant electric field, cf. equation (65), it follows

$$
a\left(k, \tau ; E_{0}\right)=\exp \left\{-\frac{\mathrm{i}}{\hbar}\left[\frac{k^{2}}{2 m_{\mathrm{e}}} \tau+\frac{e_{0}^{2} E_{0}^{2}}{24 m_{\mathrm{e}}} \tau^{3}\right]\right\} .
$$

The field dependence give rise to a nonharmonic dependence on the difference time $\tau$ which transforms to a modified nonharmonic energy spectrum. Calculating the Fourier transform of (94) yields, e.g. [6],

$$
a\left(k, \omega ; E_{0}\right)=\frac{2 \pi \hbar}{\alpha\left(E_{0}\right)} \operatorname{Ai}\left(\frac{k^{2} / 2 m_{\mathrm{e}}-\hbar \omega}{\alpha\left(E_{0}\right)}\right),
$$

where Ai is the Airy function ${ }^{13}$ and $\alpha=\left(\hbar^{2} e_{0}^{2} E_{0}^{2} / 8 m_{\mathrm{e}}\right)^{1 / 3}$. This is an exact result valid for arbitrary field strength. Its main feature is that, due to the action of the field, the sharp energy spectrum of the field-free case, equation (90), is replaced by a broadened peak, together with additional lower side peaks.

Consider now the case of a monochromatic time-dependent field, equation (66), then the solution (94) becomes

$$
a\left(k, \tau ; E_{0}, \Omega\right)=\exp \left\{-\frac{\mathrm{i}}{\hbar}\left[\left(\frac{k^{2}}{2 m_{\mathrm{e}}}+U_{\text {pond }}\right) \tau+\Delta E\left(t, \tau ; E_{0}, \Omega\right)\right]\right\},
$$

where $U_{\text {pond }} \equiv\left(\mathrm{e}^{2} E_{0}^{2}\right) /\left(4 m_{\mathrm{e}} \Omega^{2}\right)$ is the mean kinetic energy of a free particle in the field (ponderomotive energy) and the field-dependent energy correction is

$$
\Delta E\left(t, \tau ; E_{0}, \Omega\right) \equiv-U_{\text {pond }}\left(\frac{\sin \Omega \tau \cos 2 \Omega t}{\Omega}-\frac{\sin ^{2} \Omega t \sin ^{2} \Omega \tau / 2}{\Omega^{2} \tau}\right) .
$$

As discussed e.g. in [6,9], this spectral function describes an "electron-photon-quasiparticle". For example, in a harmonic field with frequency $\Omega$ and amplitude $E_{0}$, the peak in the corresponding energy spectrum is shifted from $k^{2} / 2 m_{\mathrm{e}}$ to $k^{2} / 2 m_{\mathrm{e}}+U_{\text {pond }}$, and there appear additional peaks at a distance of integer multiples of $\hbar \Omega$ from this peak.

\subsubsection{Spectral function for a weakly inhomogeneous system. Effective quantum potential}

Let us now discuss the influence of a space-dependent confinement potential $V_{I}$, first without external field $\mathbf{A}$. Then, the equation for $g^{R}$ reads

$$
\begin{aligned}
& \left\{\mathrm{i} \hbar \frac{\partial}{\partial \tau}-\frac{1}{2 m_{\mathrm{e}}}\left[\left(\frac{\hbar}{2 i} \nabla_{\mathbf{R}}\right)^{2}+k^{2}\right]\right\} g^{R}\left(\mathbf{R}, \mathbf{k}, t_{1}, t_{1}^{\prime}\right) \\
& \quad-\frac{1}{2} \int \mathrm{d}^{3} r \int \frac{\mathrm{d}^{3} k_{1}}{(2 \pi \hbar)^{3}}\left\{\mathrm{e}^{-\mathrm{i} \frac{\mathbf{r}\left(\mathbf{k}-\mathbf{k}_{1}\right)}{\hbar}} V_{I}^{\text {eff }}\left(\mathbf{R}+\frac{\mathbf{r}}{2}, t_{1}\right)+\mathrm{e}^{\mathrm{i} \frac{\mathbf{r}\left(\mathbf{k}-\mathbf{k}_{1}\right)}{\hbar}} V_{I}^{\text {eff }}\left(\mathbf{R}-\frac{\mathbf{r}}{2}, t_{1}^{\prime}\right)\right\} \\
& \quad \times \tilde{g}^{R / A}\left(\mathbf{R}, \mathbf{k}_{1}, t_{1}, t_{1}^{\prime}\right)=\delta(\tau) .
\end{aligned}
$$

${ }^{13}$ We make use of the integral representation of the Airy function

$$
\int_{0}^{\infty} \cos \left(a t^{3} \pm x t\right) \mathrm{d} t=\frac{\pi}{(3 a)^{1 / 3}} \mathrm{Ai}\left[ \pm \frac{x}{(3 a)^{1 / 3}}\right] .
$$


If the potential is weakly inhomogeneous, i.e. $\nabla_{\mathbf{R}} V_{I}^{\text {eff }}$ is small and $V_{I}^{\text {eff }}(\mathbf{R}+\mathbf{r} / 2) \approx$ $V_{I}^{\text {eff }}(\mathbf{R})$, then the solution of equation (98) is given by (for generality, we include the time-dependent Hartree mean field into the potential)

$$
a(\mathbf{R}, k, \tau, t)=\mathrm{e}^{-\frac{\mathrm{i}}{\hbar}\left[\frac{k^{2}}{2 m_{\mathrm{e}}} \tau+\int_{t-\tau / 2}^{t+\tau / 2} \mathrm{~d} \bar{t} V_{I}^{\mathrm{eff}}(\mathbf{R}, \bar{t})\right]} .
$$

It is obvious that this "local" approximation for the propagator where the electrons at each space point are assigned a definite single-particle energy is valid only in the classical limit. In contrast, for quantum particles, this is prevented by the Heisenberg uncertainty principle which has the consequence that kinetic and potential energy operators do not commute. In the following we will define an effective quantum potential $V_{Q}^{\text {eff }}[23]$ which allows us to retain the classical local form of the propagator but properly takes into account quantum diffraction effects,

$$
a(\mathbf{R}, \mathbf{k}, \tau, t)=\mathrm{e}^{-\frac{\mathrm{i}}{\hbar}\left[\frac{k^{2}}{2 m_{\mathrm{e}}} \tau+\int_{t-\tau / 2}^{t+\tau / 2} \mathrm{~d} \bar{t} V_{Q}^{\mathrm{eff}}(\mathbf{R}, \mathbf{k}, \bar{t})\right]} .
$$

This effective potential is momentum dependent and will be analyzed in section 3.9.4. For completeness, we mention that similar concepts of an effective quantum potential have been used in a variety of contexts. We mention the work of Feynman and Kleinert on the application of path integrals, e.g. [24], Kelbg and others on quantum pair potentials, cf. [34,35] and references therein and Ferry and co-workers on effective potentials for quantum transport $[25,26]$. Here, for the first time this idea is applied to the nonequilibrium spectral properties of quantum particles in a strong field. For the present application, the advantage of this definition of the quantum potential is the formal decoupling of the spectral problem from the cluster dynamics.

Finally, we restore the external electric field and assume that the same representation in form of a local spectral function is possible. In that case we have to allow for a field dependent quantum potential,

$$
a(\mathbf{R}, \mathbf{k}, \tau, t)=\mathrm{e}^{-\frac{\mathrm{i}}{\hbar}\left\{\frac{k^{2}}{2 m_{\mathrm{e}}} \tau+\int_{t-\tau / 2}^{t+\tau / 2} \mathrm{~d} \bar{t}\left[\frac{\left(\mathbf{K}^{A}\left(\bar{t}, t^{\prime}\right)\right)^{2}+\left(\mathbf{K}^{A}\left(t^{\prime}, \bar{t}\right)\right)^{2}}{4 m_{\mathrm{e}}}+V_{Q}^{\mathrm{eff}}(\mathbf{R}, \mathbf{k}, \overline{\bar{t}} ; \mathbf{A})\right]\right\}} .
$$

Naturally, equations (100) and (101) are only an ansatz. But we will demonstrate below in section (3.9.4) that this ansatz yields a closed equation for the quantum potential which can be solved in a number of important cases.

The spectral function (101) represents a far-reaching generalization of all the above special cases. Even without knowing yet the explicit analytical structure of the quantum potential, with its help all properties which are due to space-dependence of the spectral function can be analyzed straightforwardly. These properties will show up in a variety of places, including the polarization function and the local approximation to the collision integral. The most important occurences of the spectral function are combinations such as the product

$$
\begin{array}{r}
g^{R}\left(\mathbf{R}, \mathbf{k}_{1}, \tau, t\right) g^{A}\left(\mathbf{R}, \mathbf{k}_{2},-\tau, t\right)=g^{R}\left(\mathbf{R}, \mathbf{k}_{1}, \tau, t\right)\left[g^{R}\left(\mathbf{R}, \mathbf{k}_{2}, \tau, t\right)\right]^{*}= \\
=-\frac{\mathrm{i}}{\hbar} \Theta(\tau) \mathrm{e}^{-\frac{\mathrm{i}}{\hbar}\left\{\left(\frac{k_{1}^{2}}{2 m_{\mathrm{e}}}-\frac{k_{2}^{2}}{2 m_{\mathrm{e}}}\right) \tau+\int_{t-\tau / 2}^{t+\tau / 2} \mathrm{~d} \bar{t}\left[V_{Q}^{\text {eff }}\left(\mathbf{R}, \mathbf{k}_{1}, \bar{t} ; \mathbf{A}\right)-V_{Q}^{\text {eff }}\left(\mathbf{R}, \mathbf{k}_{2}, \bar{t} ; \mathbf{A}\right)\right]\right\}},
\end{array}
$$


which enters the collision integrals, see section 3.10, and the RPA polarization $[9,11]$. Further, we can compute the gradient of the propagator,

$$
\nabla_{\mathbf{R}} g^{R}(\mathbf{R}, \mathbf{k}, \tau, t)=-\frac{\mathrm{i}}{\hbar} \int_{t-\tau / 2}^{t+\tau / 2} \mathrm{~d} \bar{t} \nabla_{\mathbf{R}} V_{Q}^{\mathrm{eff}}(\mathbf{R}, \mathbf{k}, \bar{t} ; \mathbf{A}) \cdot g^{R}(\mathbf{R}, \mathbf{k}, \tau, t),
$$

which appears in the local approximation of the collision integral etc.

\subsubsection{Calculation of the Quantum potential $V_{Q}^{\text {eff }}$}

We now discuss the quantum potential more in detail, starting with the field free case. To simplify the notation we consider a potential which is only weakly time-dependent, i.e. $\tau \partial V_{I}^{\text {eff }} / \partial t \ll V_{I}^{\text {eff }}(t)$, allowing to approximate $V_{I}^{\text {eff }}(t \pm \tau / 2) \approx$ $V_{I}^{\text {eff }}(t)^{14}$. Then, the ansatz (100) simplifies to

$$
g^{R}(\mathbf{R}, \mathbf{k}, \tau, t)=-\frac{\mathrm{i}}{\hbar} \Theta(\tau) \mathrm{e}^{-\frac{\mathrm{i}}{\hbar}\left\{\frac{k^{2}}{2 m}+V_{Q}^{\mathrm{eff}}(\mathbf{R}, \mathbf{k}, t)\right\} \tau} .
$$

Here, the quantum potential still contains a possible weak (parametric) dependence on the macroscopic time which will be suppressed below to shorten the notation. Inserting this expression into equation (98), we readily obtain the equation for the quantum potential [23]

$$
\begin{gathered}
\int \frac{\mathrm{d}^{3} k_{1}}{(2 \pi \hbar)^{3}}\left\{\delta\left(\mathbf{k}-\mathbf{k}_{1}\right)\left[V_{Q}^{\mathrm{eff}}(\mathbf{R}, \mathbf{k})-\frac{1}{8 m_{\mathrm{e}}}\left(\mathrm{i} \hbar \tau \nabla_{R}^{2} V_{Q}^{\mathrm{eff}}(\mathbf{R}, \mathbf{k})+\left[\nabla_{R} V_{Q}^{\mathrm{eff}}(\mathbf{R}, \mathbf{k})\right]^{2} \tau^{2}\right)\right]\right. \\
\left.-\int \mathrm{d}^{3} r \cos \left[r \frac{\mathbf{k}-\mathbf{k}_{1}}{\hbar}\right] V_{I}^{\mathrm{eff}}\left(\mathbf{R}+\frac{\mathbf{r}}{2}, t\right)\right\} \mathrm{e}^{-\frac{\mathrm{i}}{\hbar}\left[\frac{k_{1}^{2}}{2 m_{\mathrm{e}}}+V_{Q}^{\text {eff }}\left(\mathbf{R}, \mathbf{k}_{1}\right)\right] \tau} .
\end{gathered}
$$

This is an exact equation and various analytical or numerical solution schemes are possible. Since we are interested in weakly inhomogeneous systems it is natural to develop a perturbation solution in terms of gradients of the (effective) confinement potential. The zeroth order approximation is given by the neglect of all terms involving $\nabla_{R} V_{I}^{\text {eff }}$, thus $V_{I}^{\text {eff }}(\mathbf{R}+\mathbf{r} / 2) \approx V_{I}^{\text {eff }}(\mathbf{R})$, and the $r$-integral yields $-V_{I}^{\text {eff }}(\mathbf{R})(2 \pi \hbar)^{3} \delta\left(\mathbf{k}-\mathbf{k}_{1}\right)$. In the curly brackets in (105) we are left with $\delta\left(\mathbf{k}-\mathbf{k}_{1}\right)\left[V_{Q}^{\mathrm{eff}}(\mathbf{R}, \mathbf{k})-V_{I}^{\mathrm{eff}}(\mathbf{R})\right]$, so the zeroth order result for the quantum potential is

$$
V_{Q}^{\mathrm{eff}(0)}(\mathbf{R}, \mathbf{k}, t)=V_{I}^{\mathrm{eff}}(\mathbf{R}, t)
$$

and we essentially recover the previous result of the local spectral function (99).

\footnotetext{
${ }^{14}$ This is usually fulfilled for time dependencies arising from the Hartree-Fock selfenergy, i.e. via the distribution function. A possible exception are ionization/recombination processes where the carrier density, and thus, the effective potential $V_{I}^{\text {eff }}$ may change rapidly during a short period of time.
} 
The next approximation is obtained by solving equation (105) by replacing, in all gradient terms, $V_{Q}^{\text {eff }}(\mathbf{R}, \mathbf{k}) \rightarrow V_{I}^{\text {eff }}(\mathbf{R})$ and calculating the $r$-integral in first order in gradient terms, given by

$$
-(2 \pi \hbar)^{3} \delta\left(\mathbf{k}-\mathbf{k}_{1}\right)\left[V_{I}^{\mathrm{eff}}(\mathbf{R})-\frac{\hbar^{2}}{8} \nabla_{R}^{2} V_{I}^{\mathrm{eff}} \frac{\partial^{2}}{\partial k_{1}^{2}}\right] .
$$

Carrying out the momentum differentiation on the zeroth order Green's function, we obtain the first iteration for the quantum potential

$$
\begin{aligned}
V_{Q}^{\mathrm{eff}(1)}(\mathbf{R}, \mathbf{k}, t)= & V_{Q}^{\mathrm{eff}(0)}(\mathbf{R}, t)+\mathrm{i} \frac{\hbar \tau}{4 m} \nabla_{R}^{2} V_{I}^{\mathrm{eff}}(\mathbf{R}, t) \\
& +\frac{\tau^{2}}{8 m_{\mathrm{e}}}\left[\left[\nabla_{R} V_{I}^{\mathrm{eff}}(\mathbf{R}, t)\right]^{2}+\frac{k^{2}}{m_{\mathrm{e}}} \nabla_{R}^{2} V_{I}^{\mathrm{eff}}(\mathbf{R}, t)\right],
\end{aligned}
$$

which contains, in addition to the zeroth approximation, all contributions up to second order in the gradients. The ratio of the imaginary part (second term on the r.h.s.) to the last term is of the order of $1 / \tau_{\text {cor }}$ where $\tau_{\text {cor }}$ is the correlation time (or quasi-particle life time) and can be neglected. Using (107) we obtain for the spectral function

$$
\begin{aligned}
a(\mathbf{R}, \mathbf{k}, \tau, t) & =\mathrm{e}^{-\frac{i}{\hbar}\left\{\left[\frac{k^{2}}{2 m}+V_{I}^{\mathrm{eff}}(\mathbf{R}, t)\right] \tau+\delta V_{Q}(\mathbf{R}, \mathbf{k}, t) \tau^{3}\right\}}, \\
\text { with } \quad \delta V_{Q}(\mathbf{R}, \mathbf{k}, t) & =\frac{1}{8 m_{\mathrm{e}}}\left[\left[\nabla_{R} V_{I}^{\mathrm{eff}}(\mathbf{R}, t)\right]^{2}+\frac{k^{2}}{m_{\mathrm{e}}} \nabla_{R}^{2} V_{I}^{\mathrm{eff}}(\mathbf{R}, t)\right],
\end{aligned}
$$

where, as expected, the quantum potential becomes momentum dependent. Interestingly, the inhomogeneity of the confinement field causes an anharmonic correction to the spectral function which is similar to that of a constant electric field, cf. equation (94). Again we readily obtain the energy spectrum by a Fourier transform,

$$
a(\mathbf{R}, \mathbf{k}, \omega, t)=\frac{2 \pi \hbar}{\left[3 \hbar^{2} \delta V_{Q}\right]^{1 / 3}} \operatorname{Ai}\left(\frac{k^{2} / 2 m_{\mathrm{e}}+V_{I}^{\mathrm{eff}}(\mathbf{R}, t)-\hbar \omega}{\left[3 \hbar^{2} \delta V_{Q}\right]^{1 / 3}}\right),
$$

which is shown in figure 2. As in the case of a homogeneous field, cf. equation (95), the peak of the spectral function around $\hbar \omega=k^{2} / 2 m_{\mathrm{e}}+V_{I}^{\mathrm{eff}}(\mathbf{R}, t)$ broadens, i.e. the sharp single-particle energy is smeared out (in the figure, the parameter $\alpha$ denotes $\left.\alpha \equiv\left(3 \hbar^{2} \delta V_{Q}\right)^{1 / 3}\right)$. But here this is due to spatial inhomogeneity - which directly reflects the coordinate-momentum uncertainty of quantum mechanics ${ }^{15}$. Correspondingly, with increasing inhomogeneity, the peak position shifts to higher energies, i.e. the effective local single-particle energy increases. This is readily understood: in an inhomogeneous confinement field, a quantum particle aquires an additional kinetic energy which arises from spatial compression of its wave function which is proportional to the local curvature of the field.

\footnotetext{
${ }^{15}$ In contrast to the field case, this is a perturbation result in terms of potential gradients.
} 


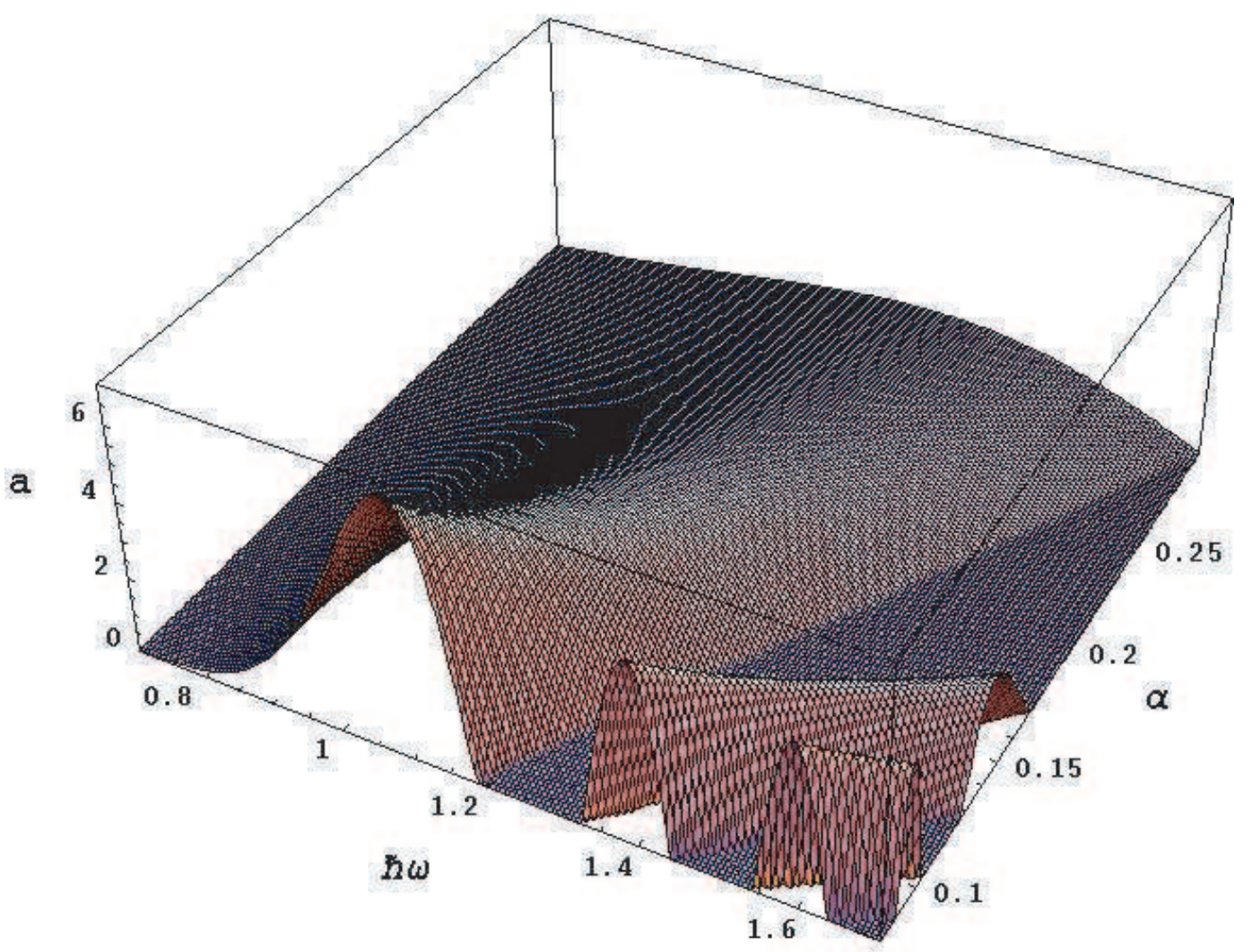

Figure 2. Spectral function $a(\mathbf{R}, \mathbf{k}, \omega, t ; \alpha)$, equation (110), at fixed time $t$ and a fixed phase space point $(\mathbf{R}, \mathbf{k})$ such that the dimensionless local single-particle energy $\epsilon(\mathbf{R}, \mathbf{k})=k^{2} / 2 m+V_{I}^{\text {eff }}(\mathbf{R}, t)=1$. Thus, the ideal spectral function would have a singularity at $\hbar \omega=1$ which is observed in the limit of vanishing inhomogeneity $\alpha \equiv\left(3 \hbar^{2} \delta V_{Q}\right)^{1 / 3} \rightarrow 0$. With increasing inhomogeneity, due to the Heisenberg uncertainty, the main peak broadens and shifts towards higher energies.

\subsubsection{Quantum potential for a system in an external field}

We now restore the electromagnetic field in our equations and generalize the result for the quantum potential. The calculations follow exactly the same lines as before, so we limit ourselves to presenting the equation for the effective quantum potential in lowest order gradient approximation

$$
\begin{aligned}
\{\mathrm{i} \hbar & \frac{\partial}{\partial \tau}+\frac{\mathrm{i} \hbar}{\tau} \mathbf{K}_{+}^{A}\left(t_{1}, t_{1}^{\prime}\right) \nabla_{\mathbf{k}}-\frac{1}{2 m_{\mathrm{e}}}\left(\frac{\hbar}{2 i} \nabla_{R}\right)^{2}+\frac{\mathrm{i} \hbar}{4 m_{\mathrm{e}}} \mathbf{K}_{-}^{A}\left(t_{1}, t_{1}^{\prime}\right) \nabla_{R} \\
& -\frac{1}{4 m_{\mathrm{e}}}\left[\left(\mathbf{k}+\mathbf{K}^{A}\left(t_{1}, t_{1}^{\prime}\right)\right)^{2}+\left(\mathbf{k}+\mathbf{K}^{A}\left(t_{1}^{\prime}, t_{1}\right)\right)^{2}\right] \\
& \left.-\left(V_{I}^{\mathrm{eff}}(\mathbf{R}, t)-\frac{\hbar^{2}}{8} \nabla_{R}^{2} V_{I}^{\mathrm{eff}}(\mathbf{R}, t) \frac{\partial^{2}}{\partial k^{2}}\right)\right\} a\left(\mathbf{R}, \mathbf{k}, t_{1}, t_{1}^{\prime}\right)=0
\end{aligned}
$$


with the ansatz for the spectral function

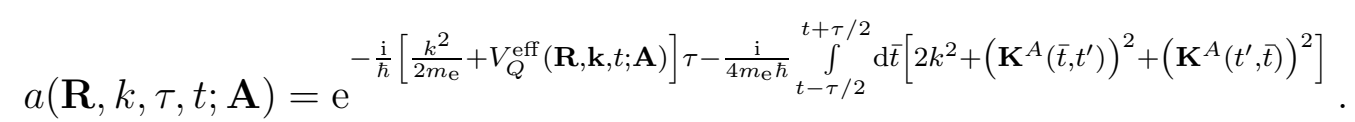

Compared to the field-free equation for the quantum potential, equation (111) contains a homogeneous electromagnetic field of arbitrary strength and time-dependence. The field couples to the space dependence of the quantum potential via the term $\mathbf{K}_{-}^{A}\left(t_{1}, t_{1}^{\prime}\right) \nabla_{R}$ giving rise to an additional contribution to the quantum potential (second term on the r.h.s.):

$$
\begin{aligned}
V_{Q}^{\mathrm{eff}(1)}(\mathbf{R}, \mathbf{k}, t ; \mathbf{A})= & V_{Q}^{\mathrm{eff}(0)}(\mathbf{R}, t)+\frac{\tau}{4 m_{\mathrm{e}}} \mathbf{K}_{-}^{A}\left(t_{1}, t_{1}^{\prime}\right) \nabla_{R} V_{I}^{\mathrm{eff}}(\mathbf{R}, t) \\
& +\frac{\tau^{2}}{8 m_{\mathrm{e}}}\left[\left[\nabla_{R} V_{I}^{\mathrm{eff}}(\mathbf{R}, t)\right]^{2}+\frac{k^{2}}{m_{\mathrm{e}}} \nabla_{R}^{2} V_{I}^{\mathrm{eff}}(\mathbf{R}, t)\right],
\end{aligned}
$$

which is proportional to the scalar product of the external field and the force acting on the electrons from the effective confinement potential. Inserting this result in equation (112) we obtain a very general result for the spectral function of electrons in a combined external electromagnetic field and weakly inhomogeneous confinement potential.

While this result represents the lowest order approximation in terms of potential gradients, it is valid for an arbitrary electromagnetic field. For special time dependencies of the field further analytical progress can be made. As an example, consider again a time-independent field. Then equation (88) yields $\mathbf{K}_{-}^{A}\left(t_{1}, t_{1}^{\prime}\right)=e_{0} \mathbf{E}_{0} \tau / 2$, and the quantum potential becomes

$$
\begin{aligned}
V_{Q}^{\mathrm{eff}(1)}(\mathbf{R}, \mathbf{k}, t ; \mathbf{A}) \tau & =V_{Q}^{\mathrm{eff}(0)}(\mathbf{R}, t) \tau+\delta V_{Q}(\mathbf{R}, \mathbf{k}, t ; \mathbf{A}) \tau^{3} \\
\delta V_{Q}(\mathbf{R}, \mathbf{k}, t ; \mathbf{A}) & =\frac{1}{8 m_{\mathrm{e}}}\left[\left[\nabla_{R} V_{I}^{\mathrm{eff}}\right]^{2}+\frac{k^{2}}{m_{\mathrm{e}}} \nabla_{R}^{2} V_{I}^{\mathrm{eff}}+e_{0} \mathbf{E}_{0} \cdot \nabla_{R} V_{I}^{\mathrm{eff}}\right]
\end{aligned}
$$

The resulting spectral function in frequency space has again the form (110) and is represented by figure 2 with $\alpha$ denoting $\left(3 \hbar^{2} \delta V_{Q}\right)^{1 / 3}$, where now $\delta V_{Q}$ has to be replaced by the generalized field dependent result (114).

\subsection{Collision integral}

In this section we derive the collision integral in the kinetic equation of the valence electrons. The main goal here is to see both the effect of the external field and the confinement potential on the collision process. An important issue is the choice of the asymptotic states of the electrons long before and after the collision process. In contrast to plasmas, here the electrons are bound to the cluster core, i.e. their wave functions are not free plane waves. The modification can be two-fold: first, the single-particle spectrum is modified and second, the asymptotic states of the scattering electron pair may be correlated. In the following, we will neglect the latter aspect, improvements can be made by adopting results from metal theory 
and compute T-matrix cross sections if necessary, e.g. [27]. The first aspect, on the other hand, is expected to be captured correctly by the electron spectral functions (or, equivalently, the propagators $g^{R / A}$ ), cf. section 3.9. Finally, the use of a static potential should be criticized as well. We expect essential modifications to arise from collective excitations (plasmons) and efficient scattering processes which involve emission and absorption of Mie plasmons by the electrons. The generalization of the collision integral to the dynamically screened Born approximation is straightforward and can follow earlier work for homogeneous plasmas in strong field [9].

Having these remarks in mind, it is reasonable to start with the simplest approximation - the static second Born approximation. The corresponding gauge-invariant expression for the selfenergy [8] in local approximation is given by

$$
\begin{aligned}
\Sigma^{\gtrless}\left(\mathbf{R}, \mathbf{k}_{1}, t, t^{\prime}\right)= & \hbar^{2} \int \frac{\mathrm{d} \mathbf{k}_{2} \mathrm{~d} \overline{\mathbf{k}}_{1} \mathrm{~d} \overline{\mathbf{k}}_{2}}{(2 \pi \hbar)^{9}} V^{2}\left(\mathbf{k}_{1}-\overline{\mathbf{k}}_{2}\right) \delta\left(\mathbf{k}_{1}+\mathbf{k}_{2}-\overline{\mathbf{k}}_{1}-\overline{\mathbf{k}}_{2}\right) \\
& \times g^{\gtrless}\left(\mathbf{R}, \overline{\mathbf{k}}_{1}, t, t^{\prime}\right) g^{\gtrless}\left(\mathbf{R}, \overline{\mathbf{k}}_{2}, t, t^{\prime}\right) g^{\lessgtr}\left(\mathbf{R}, \mathbf{k}_{2}, t^{\prime}, t\right),
\end{aligned}
$$

which, using (72), leads to the collision integral

$$
\begin{aligned}
& I\left(\mathbf{R}, \mathbf{k}_{1}, t\right)=-2 \hbar^{2} \operatorname{Re} \int \frac{\mathrm{d} \mathbf{k}_{2} \mathrm{~d} \overline{\mathbf{k}}_{1} \mathrm{~d} \overline{\mathbf{k}}_{2}}{(2 \pi \hbar)^{9}} V^{2}\left(\mathbf{k}_{1}-\overline{\mathbf{k}}_{2}\right) \delta\left(\mathbf{k}_{1}+\mathbf{k}_{2}-\overline{\mathbf{k}}_{1}-\overline{\mathbf{k}}_{2}\right) \\
& \quad \int_{t_{0}}^{t} \mathrm{~d} \bar{t}\left\{g^{>}\left(\mathbf{R}, \overline{\mathbf{k}}_{1}^{A}, t, \bar{t}\right) g^{>}\left(\mathbf{R}, \overline{\mathbf{k}}_{2}^{A}, t, \bar{t}\right) g^{<}\left(\mathbf{R}, \mathbf{k}_{2}^{A}, \bar{t}, t\right) g^{<}\left(\mathbf{R}, \mathbf{k}_{1}^{A}, \bar{t}, t\right)-(>\leftrightarrow<)\right\},
\end{aligned}
$$

where the superscript "A" denotes that all momenta are shifted, according to $\mathbf{k}_{1}^{A} \equiv$ $\mathbf{k}_{1}+\mathbf{K}^{A}(t, \bar{t})$ etc. Now we express the two-time correlation functions by the Wigner distributions using the generalized Kadanoff-Baym ansatz (74),

$$
\begin{aligned}
& I\left(\mathbf{R}, \mathbf{k}_{1}, t\right)=-2 \hbar^{2} \operatorname{Re} \int \frac{\mathrm{d} \mathbf{k}_{2} \mathrm{~d} \overline{\mathbf{k}}_{1} \mathrm{~d} \overline{\mathbf{k}}_{2}}{(2 \pi \hbar)^{9}} V^{2}\left(\mathbf{k}_{1}-\overline{\mathbf{k}}_{2}\right) \delta\left(\mathbf{k}_{1}+\mathbf{k}_{2}-\overline{\mathbf{k}}_{1}-\overline{\mathbf{k}}_{2}\right) \\
& \quad \times \int_{t_{0}}^{t} \mathrm{~d} \bar{t} g^{R}\left(\mathbf{R}, \overline{\mathbf{k}}_{1}^{A}, t, \bar{t}\right) g^{R}\left(\mathbf{R}, \overline{\mathbf{k}}_{2}^{A}, t, \bar{t}\right)\left[g^{R}\left(\mathbf{R}, \mathbf{k}_{2}^{A}, t, \bar{t}\right)\right]^{*}\left[g^{R}\left(\mathbf{R}, \mathbf{k}_{1}^{A}, t, \bar{t}\right)\right]^{*} \\
& \quad \times\left\{f^{>}\left(\mathbf{R}, \overline{\mathbf{k}}_{1}^{Q}, \bar{t}\right) f^{>}\left(\mathbf{R}, \overline{\mathbf{k}}_{2}^{Q}, \bar{t}\right) f^{<}\left(\mathbf{R}, \mathbf{k}_{2}^{Q}, \bar{t}\right) f^{<}\left(\mathbf{R}, \mathbf{k}_{1}^{Q}, \bar{t}\right)-(>\leftrightarrow<)\right\},
\end{aligned}
$$

where the shift of the momentum arguments in the distribution functions is now given by $\mathbf{Q}_{A}$, equation (127) in the Appendix, i.e. $\mathbf{k}_{1}^{Q} \equiv \mathbf{k}_{1}+\mathbf{Q}_{A}(t, \bar{t})$ and so on. What is left now is to evaluate the spectral information of the four propagators which determines the energy balance of the scattering event in the combined external and confinement fields. Using the result for the product of two propagators, equation (102), we immediately obtain

$$
\begin{aligned}
& I\left(\mathbf{R}, \mathbf{k}_{1}, t\right)=-2 \int \frac{\mathrm{d} \mathbf{k}_{2} \mathrm{~d} \overline{\mathbf{k}}_{1} \mathrm{~d} \overline{\mathbf{k}}_{2}}{(2 \pi \hbar)^{9}} V^{2}\left(\mathbf{k}_{1}-\overline{\mathbf{k}}_{2}\right) \delta\left(\mathbf{k}_{1}+\mathbf{k}_{2}-\overline{\mathbf{k}}_{1}-\overline{\mathbf{k}}_{2}\right) \\
& \quad \times \int_{t_{0}}^{t} \mathrm{~d} \bar{t} \cos \left\{\frac{1}{\hbar} \int_{t_{0}}^{t-\bar{t}} \mathrm{~d} t_{1}\left[\epsilon\left(\mathbf{R}, \overline{\mathbf{k}}_{1}, t_{1}\right)+\epsilon\left(\mathbf{R}, \overline{\mathbf{k}}_{2}, t_{1}\right)-\epsilon\left(\mathbf{R}, \mathbf{k}_{1}, t_{1}\right)-\epsilon\left(\mathbf{R}, \mathbf{k}_{2}, t_{1}\right)\right]\right\} \\
& \quad \times\left\{f^{>}\left(\mathbf{R}, \overline{\mathbf{k}}_{1}^{Q}, \bar{t}\right) f^{>}\left(\mathbf{R}, \overline{\mathbf{k}}_{2}^{Q}, \bar{t}\right) f^{<}\left(\mathbf{R}, \mathbf{k}_{2}^{Q}, \bar{t}\right) f^{<}\left(\mathbf{R}, \mathbf{k}_{1}^{Q}, \bar{t}\right)-(>\leftrightarrow<)\right\},
\end{aligned}
$$


where the effective single-particle energy which enters the energy balance is given by

$$
\epsilon(\mathbf{R}, \mathbf{k}, t) \equiv \frac{k^{2}}{2 m_{\mathrm{e}}}+V_{Q}^{\mathrm{eff}}(\mathbf{R}, \mathbf{k}, t ; \mathbf{A})
$$

As in the homogeneous case, the field drops out of the energy balance of scattering of particles with same charge to mass ratio since the field does not change their distance. In contrast, in the case of electron ion scattering the field changes the energy balance by [8] $\left(\overline{\mathbf{k}}_{1}-\mathbf{k}_{1}\right) \mathbf{R}_{A}(t, \bar{t})$, where $\mathbf{R}_{A}$ here is the distance change of two particles in the electromagnetic field which is defined in analogy to definition (128). If the ions are treated as a fixed background (as it is the case with the jellium model), no electron-ion collision integral appears; electron-ion scattering then appears via the jellium potential on the left hand side of the kinetic equation. The corresponding nonlinear effects in a strong field are analyzed in [11].

We see from equation (118) that the confinement field does in fact have an influence on the scattering process which arises from the momentum dependence of the quantum potential. This is directly seen from the explicit result for the quantum potential, equation (113). Using this result, we can rewrite the energy difference $\Delta \epsilon$ in equation (118) and see that the last term in the quantum potential (113) modifies the energy balance to

$$
\begin{aligned}
& \Delta \epsilon_{12}\left(\mathbf{R}, \mathbf{k}_{1}, \mathbf{k}_{2}, \overline{\mathbf{k}}_{1}, \overline{\mathbf{k}}_{2}, t ; \mathbf{A}\right) \equiv \\
& \quad \equiv \epsilon\left(\mathbf{R}, \mathbf{k}_{1}, t ; \mathbf{A}\right)+\epsilon\left(\mathbf{R}, \mathbf{k}_{2}, t ; \mathbf{A}\right)-\epsilon\left(\mathbf{R}, \overline{\mathbf{k}}_{1}, t ; \mathbf{A}\right)-\epsilon\left(\mathbf{R}, \overline{\mathbf{k}}_{2}, t ; \mathbf{A}\right)= \\
& \quad=\Delta E_{12}\left[1+\frac{\tau^{2}}{4 m_{\mathrm{e}}} \nabla^{2} V_{I}^{\mathrm{eff}}(\mathbf{R}, t)\right]
\end{aligned}
$$

where $\Delta E_{12}=\left(k_{1}^{2}+k_{2}^{2}-\bar{k}_{1}^{2}-\bar{k}_{2}^{2}\right) / 2 m_{\mathrm{e}}$. We see that the difference of quasiparticle energies (120) contains, in addition to the difference of kinetic energies of the particle pair, a term proportional to the local curvature of the effective confinement potential.

To verify if this has a consequence on the energy balance in a two-particle collision we consider the Markov limit of the collision integral in Born approximation, equation (118): neglecting the time dependence of the distribution functions compared to the correlation time and extending the $\bar{t}$-integration to infinity the integration can be performed using (96). As a result the energy kernel of the collision integral becomes

$$
\frac{\pi\left(4 m_{\mathrm{e}}\right)^{1 / 3}}{\left[\Delta E_{12} \nabla^{2} V_{I}^{\text {eff }}\right]} \operatorname{Ai}\left[\left(\frac{4 m_{\mathrm{e}}\left(\Delta E_{12}\right)^{2}}{\nabla^{2} V_{I}^{\mathrm{eff}}}\right)^{1 / 3}\right] \text {. }
$$

This function is singular at $\Delta E_{12}=0$, i.e. the dominant spectral weight falls on scattering processes which conserve the kinetic energy of the particle pair, as in the case of a homogeneous system (in the Markov limit). The latter case is recovered by the limit $\nabla V_{I}^{\text {eff }} \rightarrow 0$ and leads to the familiar result $\pi \delta\left(\Delta E_{12}\right)$. A larger effect of the inhomogeneity on the scattering process occurs on short time scales of the 
order of the correlation time where the Markov limit fails, e.g. [2]. Then the energy broadening arising from the finite collision duration is additionally increased due to the inhomogeneity of the confinement field.

Aside from electron-electron scattering, there are numerous physical situations where the presence of an inhomogeneous field will have an even more pronounced effect on the microscopic scattering probability. The most important one is inelastic scattering. Indeed if particles, after the collision appear in a different quantum state (energy level or band) with a different dispersion (effective mass), the quantum potential will be different before and after the collision, even if the external field is independent of the quantum state. This effect should be directly observable in confined quantum systems undergoing e.g. collisional excitation or ionization.

\section{Discussion}

In this paper a gauge-invariant nonequilibrium Green's functions theory for weakly inhomogeneous systems has been developed. Weak inhomogeneity covers a broad class of many-particle systems of current interest, including electrons in quantum dots, ultracold ions in traps, valence electrons in metal clusters and so on. These systems are conveniently treated within the Wigner representation by a direct extension of the quantum kinetic theory for spatially homogeneous systems by including spatial gradient corrections. Here, we derived the corresponding Kadanoff-Baym/Keldysh equations for the two-time correlation functions including an arbitrary (homogeneous) strong time-dependent electromagnetic field and a weakly inhomogeneous confinement potential. From the KBE the gauge-invariant equations for the Wigner distribution and for the retarded Green's function (propagator) have been obtained.

Special attention has been devoted to an analysis of the spectral properties of the electrons. Introducing the concept of an effective quantum potential which replaces the effective (mean-field) potential $V_{I}^{\text {eff }}$, the spectral function of electrons in a combined electromagnetic field and confinement field has been derived. Its main characteristics is that, even without correlations, the spectral function is broadened and blue-shifted as a result of kinetic energy gain of the electrons from the electromagnetic field as well as from spatial localization (wave function compression) in the confinement field. This has direct consequences for scattering processes and modifies the energy balance and thus, the collision cross section and scattering rates.

Further applications of the results of the present theory to solutions of the quantum kinetic equation in linear response, to the dielectric properties and to solutions in a strong field are straightforward and will be presented in a separate publication [11]. 


\section{Appendix: Gauge invariant Kadanoff-Baym/Keldysh equations for correlated electrons in a strong electromagnetic field and inhomogeneous external potential}

In this appendix we present details of the derivation of the gauge-invariant Kadanoff-Baym/Keldysh equations (KBE) in Wigner representation, the equations for the Wigner function $f(\mathbf{R}, \mathbf{k}, t)$ and for the retarded and advanced Green's function (propagators) $g^{R / A}\left(\mathbf{R}, \mathbf{k}, t, t^{\prime}\right)$. The derivation is kept as general as possible. In particular, we allow for an arbitrary single-particle energy dispersion $\epsilon(\mathbf{k})$, thus, making the results applicable to electrons in condensed matter systems as well. The results of the main text follow by using the limit of parabolic dispersion $\epsilon(\mathbf{k})=k^{2} / 2 m_{\mathrm{e}}$.

The KBE in the presence of a strong homogeneous electric field and an inhomogeneous confinement potential $V_{I}$ read, in coordinate space,

$$
\begin{aligned}
& {\left[\mathrm{i} \hbar \frac{\partial}{\partial t_{1}}-\epsilon\left(\frac{\hbar}{\mathrm{i}} \nabla_{1}+\frac{e_{0}}{c} \mathbf{A}(1)\right)-V_{I}(1)\right] g^{\gtrless}\left(1,1^{\prime}\right)=\int \mathrm{d} \overline{\mathbf{r}}_{1} \Sigma^{\mathrm{HF}}\left(1, \overline{\mathbf{r}}_{1} t_{1}\right) g^{\gtrless}\left(\overline{\mathbf{r}}_{1} t_{1}, 1^{\prime}\right)} \\
& \quad+\int_{t_{0}}^{t_{1}} \mathrm{~d} \overline{1}\left[\Sigma^{>}(1, \overline{1})-\Sigma^{<}(1, \overline{1})\right] g^{\gtrless}\left(\overline{1}, 1^{\prime}\right)-\int_{t_{0}}^{t_{1}^{\prime}} \mathrm{d} \overline{1} \Sigma^{\gtrless}(1, \overline{1})\left[g^{>}\left(\overline{1}, 1^{\prime}\right)-g^{<}\left(\overline{1}, 1^{\prime}\right)\right],
\end{aligned}
$$

where we denoted $1 \equiv\left(\mathbf{r}_{1}, t_{1}, s_{1}^{3}\right)$. We now derive the gauge-invariant Wigner representation of this equation. For this we introduce macroscopic and microscopic length scales, $\mathbf{R} \equiv\left(\mathbf{r}_{1}+\mathbf{r}_{1}\right) / 2$ and $\mathbf{r} \equiv \mathbf{r}_{1}-\mathbf{r}_{2}$, where the first is determined by the geometry of the confinement potential $V_{I}$. Then, the gradient in the momentum operator in equation (50) becomes $\nabla_{r_{1}}=1 / 2 \nabla_{R}+\nabla_{r}$. We now apply the spatial part of the transform (58) to the 1.h.s. of equation (122),

$$
\begin{gathered}
\int \mathrm{d}^{3} r \mathrm{e}^{-\frac{\mathrm{i}}{\hbar} \mathbf{r}\left[\mathbf{k}-\frac{e_{0}}{c} \int_{t_{1}^{\prime}}^{t_{1}} \mathrm{~d} \bar{t} \frac{\mathbf{A}(\bar{t})}{t_{1}-t_{1}^{\prime}}\right]}\left[\mathrm{i} \hbar \frac{\partial}{\partial t_{1}}-\epsilon\left(\frac{\hbar}{\mathrm{i}} \nabla_{r}+\frac{1}{2} \frac{\hbar}{i} \nabla_{R}+\frac{e_{0}}{c} \mathbf{A}(1)\right)-V_{I}(1)\right] \\
\times \int \frac{\mathrm{d}^{3} k_{1}}{(2 \pi \hbar)^{3}} \mathrm{e}^{\frac{\mathrm{i}}{\hbar} \mathbf{r}\left[\mathbf{k}_{1}-\frac{e_{0}}{c} \int_{t_{1}^{\prime}}^{t_{1}} \mathrm{~d} \bar{t} \frac{\mathbf{A}(\bar{t})}{t_{1}-t_{1}^{\prime}}\right]} \tilde{g} \gtrless\left(\mathbf{R}, \mathbf{k}_{1}, t_{1}, t_{1}^{\prime}\right),
\end{gathered}
$$

where the tilde denotes the Wigner transformed functions. Performing the $t_{1}$ and $r$ derivatives on the second exponential and noticing that the vector potential terms in the exponentials cancel, we obtain from equation (123)

$$
\begin{array}{r}
\int \mathrm{d}^{3} r \int \frac{\mathrm{d}^{3} k_{1}}{(2 \pi \hbar)^{3}} \mathrm{e}^{-\frac{\mathrm{i}}{\hbar} \mathbf{r}\left(\mathbf{k}-\mathbf{k}_{1}\right)}\left\{\left[\mathrm{i} \hbar \frac{\partial}{\partial t_{1}}-\mathbf{r} \frac{\mathbf{K}^{A}\left(t_{1}, t_{1}^{\prime}\right)}{t_{1}-t_{1}^{\prime}}-V_{I}(1)\right]\right. \\
\left.-\epsilon\left[\frac{1}{2} \frac{\hbar}{\mathrm{i}} \nabla_{R}+\mathbf{k}_{1}-\mathbf{K}^{A}\left(t_{1}, t_{1}^{\prime}\right)\right]\right\} \tilde{g}^{\gtrless}\left(\mathbf{R}, \mathbf{k}_{1}, t_{1}, t_{1}^{\prime}\right),
\end{array}
$$

where we introduced the definition

$$
\mathbf{K}^{A}\left(t, t^{\prime}\right) \equiv-\frac{e_{0}}{c} \int_{t^{\prime}}^{t} \mathrm{~d} t^{\prime \prime} \frac{\mathbf{A}(t)-\mathbf{A}\left(t^{\prime \prime}\right)}{t-t^{\prime}}
$$


with the properties $\mathbf{K}^{A}(t, t)=0$ and

$$
\mathbf{K}^{A}\left(t, t^{\prime}\right)-\mathbf{K}^{A}\left(t^{\prime}, t\right)=-\frac{e_{0}}{c}\left\{\mathbf{A}(t)-\mathbf{A}\left(t^{\prime}\right)\right\}=e_{0} \int_{t^{\prime}}^{t} \mathrm{~d} t^{\prime \prime} \mathbf{E}\left(t^{\prime \prime}\right) .
$$

Further, we note the relation of $\mathbf{K}^{A}$ to the momentum gain and displacement of a free particle in the electromagnetic field, within the time interval $\left[t^{\prime}, t\right]$,

$$
\begin{aligned}
& \mathbf{Q}_{A}\left(t, t^{\prime}\right)=\mathbf{K}^{A}\left(t, t^{\prime}\right)-\mathbf{K}^{A}\left(t^{\prime}, t\right) \\
& \mathbf{R}_{A}\left(t, t^{\prime}\right)=-\frac{1}{m_{\mathrm{e}}} \mathbf{K}^{A}\left(t, t^{\prime}\right)\left(t-t^{\prime}\right) .
\end{aligned}
$$

Using $\mathbf{r}=\hbar / i \nabla_{\mathbf{k}_{1}}$, the $r$-integration can be performed ${ }^{16}$, giving $\mathbf{k}_{1}=\mathbf{k}$, except for the potential term where the center of mass and relative coordinates, in general, do not separate. We obtain the gauge-invariant kinetic equation in Wigner representation

$$
\begin{aligned}
\left\{\left[\mathrm{i} \hbar \frac{\partial}{\partial t_{1}}+\right.\right. & \left.\left.\mathrm{i} \hbar \frac{\mathbf{K}^{A}\left(t_{1}, t_{1}^{\prime}\right)}{t_{1}-t_{1}^{\prime}} \nabla_{\mathbf{k}}\right]-\epsilon\left[\frac{1}{2} \frac{\hbar}{\mathrm{i}} \nabla_{R}+\mathbf{k}+\mathbf{K}^{A}\left(t_{1}, t_{1}^{\prime}\right)\right]\right\} \tilde{g}^{\gtrless}\left(\mathbf{R}, \mathbf{k}, t_{1}, t_{1}^{\prime}\right) \\
& -\int \mathrm{d}^{3} r \int \frac{\mathrm{d}^{3} k_{1}}{(2 \pi \hbar)^{3}} \mathrm{e}^{-\frac{\mathrm{i}}{\hbar} \mathbf{r}\left(\mathbf{k}-\mathbf{k}_{1}\right)} V_{I}^{\text {eff }}\left(\mathbf{R}+\frac{\mathbf{r}}{2}\right) \tilde{g}^{\gtrless}\left(\mathbf{R}, \mathbf{k}_{1}, t_{1}, t_{1}^{\prime}\right)= \\
= & \tilde{I}_{\mathrm{F}}^{\gtrless}\left(\mathbf{R}, \mathbf{k}, t_{1}, t_{1}^{\prime}\right)+\tilde{I}^{\gtrless}\left(\mathbf{R}, \mathbf{k}, t_{1}, t_{1}^{\prime}\right),
\end{aligned}
$$

where the Hartree mean field is local (momentum independent) and, therefore, can be included into the effective confinement potential,

$$
V_{I}^{\mathrm{eff}}(\mathbf{R}, t) \equiv V_{I}(\mathbf{R}, t)+\Sigma^{\mathrm{H}}(\mathbf{R}, t),
$$

and $\Sigma^{\mathrm{H}}(\mathbf{R}, t)$ is related to the definition (51) by

$$
\begin{aligned}
\Sigma^{\mathrm{H}}\left(\mathbf{r}_{1}, t_{1} ; \mathbf{r}_{1}^{\prime}, t_{1}^{\prime}\right) & =\delta\left(\mathbf{r}_{1}-\mathbf{r}_{1}^{\prime}\right) \delta\left(t_{1}-t_{1}^{\prime}\right) \Sigma^{\mathrm{H}}\left(\mathbf{r}_{1}, t_{1}\right), \\
\Sigma^{\mathrm{H}}\left(\mathbf{r}_{1}, t_{1}\right) & =\int \mathrm{d}^{3} r_{2} V\left(\mathbf{r}_{1}-\mathbf{r}_{2}\right) f\left(r_{2}, r_{2}, t\right)=\int \mathrm{d}^{3} r_{2} V\left(\mathbf{r}_{1}-\mathbf{r}_{2}\right) n\left(r_{2}, t\right) .
\end{aligned}
$$

The r.h.s. of equation (129) comprises the remaining two-particle contributions and all higher order correlations - the exchange ("F") and correlation (collision, denoted by "२") terms.

To obtain the equation for the single-time distribution function and for the propagator, we also need the adjoint equation,

$$
\begin{gathered}
\left\{\left[-\mathrm{i} \hbar \frac{\partial}{\partial t_{1}^{\prime}}+\mathrm{i} \hbar \frac{\mathbf{K}^{A}\left(t_{1}^{\prime}, t_{1}\right)}{t_{1}-t_{1}^{\prime}} \nabla_{\mathbf{k}}\right]-\epsilon\left[-\frac{1}{2} \frac{\hbar}{\mathrm{i}} \nabla_{R}+\mathbf{k}+\mathbf{K}^{A}\left(t_{1}^{\prime}, t_{1}\right)\right]\right\} \tilde{g} \gtrless\left(\mathbf{R}, \mathbf{k}, t_{1}, t_{1}^{\prime}\right) \\
-\int \mathrm{d}^{3} r \int \frac{\mathrm{d}^{3} k_{1}}{(2 \pi \hbar)^{3}} \mathrm{e}^{+\frac{\mathrm{i}}{\hbar} \mathbf{r}\left(\mathbf{k}-\mathbf{k}_{1}\right)} V_{I}\left(\mathbf{R}+\frac{\mathbf{r}}{2}\right) \tilde{g}^{\gtrless}\left(\mathbf{R}, \mathbf{k}_{1}, t_{1}, t_{1}^{\prime}\right)= \\
=-\left[\tilde{I}^{\gtrless}\left(\mathbf{R}, \mathbf{k}, t_{1}^{\prime}, t_{1}\right)\right]^{*}-\left[\tilde{I}_{\mathrm{F}}^{\gtrless}\left(\mathbf{R}, \mathbf{k}, t_{1}^{\prime}, t_{1}\right)\right]^{*},
\end{gathered}
$$

\footnotetext{
${ }^{16}$ The $r$-integration can be extended to infinity as the electron density outside the cluster is zero.
} 
where in the final expression we exchanged $t_{1} \leftrightarrow t_{1}^{\prime}$ and used the property $\left[g^{\gtrless}\left(t, t^{\prime}\right)\right]^{*}=$ $-g \gtrless\left(t^{\prime}, t\right)$.

Consider now the exchange term $\tilde{I}_{\mathrm{F}}^{\gtrless}$. The gauge-invariant Fourier transform of the coordinate space definition (51) is readily computed. Due to the locality in time of $\Sigma^{\mathrm{F}}$

$$
\begin{aligned}
\Sigma^{\mathrm{F}}\left(\mathbf{r}_{1}, t_{1} ; \mathbf{r}_{1}^{\prime}, t_{1}^{\prime}\right) & =\delta\left(t_{1}-t_{1}^{\prime}\right) \Sigma^{\mathrm{F}}\left(\mathbf{r}_{1}, \mathbf{r}_{1}^{\prime}, t_{1}\right), \\
\Sigma^{\mathrm{F}}\left(\mathbf{r}_{1}, \mathbf{r}_{1}^{\prime}, t\right) & =-V\left(\mathbf{r}_{1}-\mathbf{r}_{1}^{\prime}\right) f\left(\mathbf{r}_{1}, \mathbf{r}_{1}^{\prime}, t\right),
\end{aligned}
$$

the external field drops out of the Fourier transform $\left(K^{A}(t, t)=0\right)$,

$$
\Sigma^{\mathrm{F}}(\mathbf{R}, \mathbf{k}, t)=-\int \frac{\mathrm{d}^{3} k_{2}}{(2 \pi \hbar)^{3}} V\left(k_{2}\right) f\left(\mathbf{R}, \mathbf{k}-\mathbf{k}_{2}, t\right) .
$$

Thus the result for $\tilde{I}_{\mathrm{F}}^{\gtrless}$, up to first order gradient corrections, is given by

$$
\begin{aligned}
\tilde{I}_{\mathbf{F}}^{\gtrless}\left(\mathbf{R}, \mathbf{k}, t, t^{\prime}\right)= & {\left.\left[1+\frac{\mathrm{i} \hbar}{2}\left(\nabla_{\mathbf{R}_{1}} \nabla_{\mathbf{Q}_{2}}-\nabla_{\mathbf{R}_{2}} \nabla_{\mathbf{Q}_{1}}\right)\right] \Sigma^{F}\left(\mathbf{R}_{1}, \mathbf{Q}_{1}, t\right) g^{\gtrless}\left(\mathbf{R}_{2}, \mathbf{Q}_{2}, t, t^{\prime}\right)\right|_{(\mathbf{R}, \mathbf{k})} } \\
= & \int \frac{\mathrm{d}^{3} k_{1}}{(2 \pi \hbar)^{3}} V\left(k_{1}\right)\left\{\nabla_{\mathbf{R}_{1}} f\left(\mathbf{R}_{1}, \mathbf{Q}_{1}-\mathbf{k}_{1}, t\right) \nabla_{\mathbf{Q}_{2}} f\left(\mathbf{R}_{2}, \mathbf{Q}_{2}, t\right)\right. \\
& \left.-\nabla_{\mathbf{Q}_{1}} f\left(\mathbf{R}_{1}, \mathbf{Q}_{1}-\mathbf{k}_{1}, t\right) \nabla_{\mathbf{R}_{2}} f\left(\mathbf{R}_{2}, \mathbf{Q}_{2}, t\right)\right\}\left.\right|_{(\mathbf{R}, \mathbf{k})},
\end{aligned}
$$

where we introduced the short notation $(\mathbf{R}, \mathbf{k})$ to indicate that, after differentiation, one has to set $\mathbf{R}_{1}=\mathbf{R}_{2}=\mathbf{R}$ and $\mathbf{Q}_{1}=\mathbf{Q}_{2}=\mathbf{k}$.

The corresponding term in the adjoint equation (131) is

$$
\begin{aligned}
-\left[\tilde{I}_{\mathrm{F}}^{\gtrless}\left(\mathbf{R}, \mathbf{k}, t^{\prime}, t\right)\right]^{*}= & -\left[1-\frac{\mathrm{i} \hbar}{2}\left(\nabla_{\mathbf{R}_{1}} \nabla_{\mathbf{Q}_{2}}-\nabla_{\mathbf{R}_{2}} \nabla_{\mathbf{Q}_{1}}\right)\right] \Sigma^{F}\left(\mathbf{R}_{1}, \mathbf{Q}_{1}, t^{\prime}\right) \\
& \times\left.\left[g \gtrless\left(\mathbf{R}_{2}, \mathbf{Q}_{2}, t^{\prime}, t\right)\right]^{*}\right|_{(\mathbf{R}, \mathbf{k})} .
\end{aligned}
$$

In the kinetic equation for the Wigner function the Fock terms appear in the combination

$$
I^{\mathrm{F}}(\mathbf{R}, \mathbf{k}, t)=-2 \operatorname{Re}\left[\Sigma^{\mathrm{F}}(\mathbf{R}, \mathbf{k}, t) g^{<}(\mathbf{R}, \mathbf{k}, t, t)\right],
$$

which vanishes exactly (on the time-diagonal, $g^{<}$is purely imaginary). This means that the only nonzero exchange contributions arise from the gradient corrections which appear due to the product $\Sigma^{\mathrm{F}} \cdot g^{<}$. Using the general expression (45), we obtain

$$
\begin{aligned}
I^{\mathrm{F}}(\mathbf{R}, \mathbf{k}, t)= & \left.2 \operatorname{Re}\left(1+\frac{\mathrm{i} \hbar}{2}\{\ldots\}\right) \Sigma^{F}\left(\mathbf{R}_{1}, \mathbf{Q}_{1}, t\right) g^{<}\left(\mathbf{R}_{2}, \mathbf{Q}_{2}, t, t\right)\right|_{(\mathbf{R}, \mathbf{k})} \\
= & -\left.\left(\nabla_{\mathbf{R}_{1}} \nabla_{\mathbf{Q}_{2}}-\nabla_{\mathbf{R}_{2}} \nabla_{\mathbf{Q}_{1}}\right) \Sigma^{F}\left(\mathbf{R}_{1}, \mathbf{Q}_{1}, t\right) f\left(\mathbf{R}_{2}, \mathbf{Q}_{2}, t\right)\right|_{(\mathbf{R}, \mathbf{k})} \\
= & \int \frac{\mathrm{d}^{3} k_{1}}{(2 \pi \hbar)^{3}} V\left(k_{1}\right)\left\{\nabla_{\mathbf{R}_{1}} f\left(\mathbf{R}_{1}, \mathbf{Q}_{1}-\mathbf{k}_{1}, t\right) \nabla_{\mathbf{Q}_{2}} f\left(\mathbf{R}_{2}, \mathbf{Q}_{2}, t\right)\right. \\
& \left.-\nabla_{\mathbf{Q}_{1}} f\left(\mathbf{R}_{1}, \mathbf{Q}_{1}-\mathbf{k}_{1}, t\right) \nabla_{\mathbf{R}_{2}} f\left(\mathbf{R}_{2}, \mathbf{Q}_{2}, t\right)\right\}\left.\right|_{(\mathbf{R}, \mathbf{k})} .
\end{aligned}
$$


Now we calculate the sum and difference of the two Kadanoff-Baym/Keldysh equations in Wigner representation, equations (129), (131)

$$
\begin{aligned}
\{\mathrm{i} \hbar & {\left[\frac{\partial}{\partial t_{1}} \mp \frac{\partial}{\partial t_{1}^{\prime}}\right]+2 \mathrm{i} \hbar \frac{\mathbf{K}_{ \pm}^{A}\left(t_{1}, t_{1}^{\prime}\right)}{t_{1}-t_{1}^{\prime}} \nabla_{\mathbf{k}}-\epsilon\left[\frac{1}{2} \frac{\hbar}{\mathrm{i}} \nabla_{\mathbf{R}}+\mathbf{k}+\mathbf{K}^{A}\left(t_{1}, t_{1}^{\prime}\right)\right] } \\
\mp \epsilon & {\left.\left[-\frac{1}{2} \frac{\hbar}{\mathrm{i}} \nabla_{\mathbf{R}}+\mathbf{k}+\mathbf{K}^{A}\left(t_{1}^{\prime}, t_{1}\right)\right]\right\} \tilde{g}^{\gtrless}\left(\mathbf{R}, \mathbf{k}, t_{1}, t_{1}^{\prime}\right) } \\
& -\int \mathrm{d}^{3} r \int \frac{\mathrm{d}^{3} k_{1}}{(2 \pi \hbar)^{3}}\left\{\mathrm{e}^{-\frac{\mathrm{i}}{\hbar} \mathbf{r}\left(\mathbf{k}-\mathbf{k}_{1}\right)} V_{I}^{\mathrm{eff}}\left(\mathbf{R}+\frac{\mathbf{r}}{2}, t_{1}\right) \pm \mathrm{e}^{\frac{\mathrm{i}}{\hbar} \mathbf{r}\left(\mathbf{k}-\mathbf{k}_{1}\right)} V_{I}^{\mathrm{eff}}\left(\mathbf{R}-\frac{\mathbf{r}}{2}, t_{1}^{\prime}\right)\right\} \\
& \times \tilde{g}^{\gtrless}\left(\mathbf{R}, \mathbf{k}_{1}, t_{1}, t_{1}^{\prime}\right)=I_{\mp}^{\gtrless}\left(\mathbf{R}, \mathbf{k}, t_{1}, t_{1}^{\prime}\right),
\end{aligned}
$$

where $I_{ \pm}^{\gtrless}\left(\mathbf{R}, \mathbf{k}, t_{1}, t_{1}^{\prime}\right) \equiv \tilde{I}^{\gtrless}\left(\mathbf{R}, \mathbf{k}, t_{1}, t_{1}^{\prime}\right) \pm\left[\tilde{I}^{\gtrless}\left(\mathbf{R}, \mathbf{k}, t_{1}^{\prime}, t_{1}\right)\right]^{*}$, and $\mathbf{K}_{ \pm}^{A}$ are short notations for the sum and difference of the field-induced momenta

$$
\mathbf{K}_{ \pm}^{A}\left(t_{1}, t_{1}^{\prime}\right) \equiv \frac{1}{2}\left[\mathbf{K}^{A}\left(t_{1}, t_{1}^{\prime}\right) \pm \mathbf{K}^{A}\left(t_{1}^{\prime}, t_{1}\right)\right]
$$

To obtain the equation for the Wigner distribution, we need the difference (lower signs) and, for simplification of the notation, we, from now on, specify to a parabolic energy dispersion, $\epsilon(k) \rightarrow k^{2} / 2 m$ :

$$
\begin{aligned}
& \left\{\mathrm{i} \hbar \frac{\partial}{\partial t}+2 \mathrm{i} \hbar \frac{\mathbf{K}_{-}^{A}\left(t_{1}, t_{1}^{\prime}\right)}{t_{1}-t_{1}^{\prime}} \nabla_{\mathbf{k}}-\frac{\hbar}{\mathrm{i}} \frac{\mathbf{k}}{m_{\mathrm{e}}} \nabla_{\mathbf{R}}-\frac{1}{2 m_{\mathrm{e}}}\left[\left(\mathbf{K}^{A}\right)^{2}\left(t_{1}, t_{1}^{\prime}\right)-\left(\mathbf{K}^{A}\right)^{2}\left(t_{1}^{\prime}, t_{1}\right)\right.\right. \\
& \left.\left.\quad+4 \mathbf{k} \mathbf{K}_{-}^{A}\left(t_{1}, t_{1}^{\prime}\right)-\frac{2 \hbar}{\mathrm{i}} \mathbf{K}_{+}^{A}\left(t_{1}, t_{1}^{\prime}\right) \nabla_{\mathbf{R}}\right]\right\} \tilde{g}^{\gtrless}\left(\mathbf{R}, \mathbf{k}, t_{1}, t_{1}^{\prime}\right) \\
& \quad-\int \mathrm{d}^{3} r \int \frac{\mathrm{d}^{3} k_{1}}{(2 \pi \hbar)^{3}}\left\{\mathrm{e}^{-\frac{\mathrm{i}}{\hbar} \mathbf{r}\left(\mathbf{k}-\mathbf{k}_{1}\right)} V_{I}^{\mathrm{eff}}\left(\mathbf{R}+\frac{\mathbf{r}}{2}, t_{1}\right)-\mathrm{e}^{\frac{\mathrm{i}}{\hbar} \mathbf{r}\left(\mathbf{k}-\mathbf{k}_{1}\right)} V_{I}^{\mathrm{eff}}\left(\mathbf{R}-\frac{\mathbf{r}}{2}, t_{1}^{\prime}\right)\right\} \\
& \quad \times \tilde{g}^{\gtrless}\left(\mathbf{R}, \mathbf{k}_{1}, t_{1}, t_{1}^{\prime}\right)=I_{+}^{\gtrless}\left(\mathbf{R}, \mathbf{k}, t_{1}, t_{1}^{\prime}\right) .
\end{aligned}
$$

Finally, the kinetic equation for the Wigner function $f$ is obtained by taking equation (140) for the function $g^{<}$at equal times, $t_{1}=t_{1}^{\prime}=t$, and further expressing $g^{<}$by $f$, using relation (47)

$$
\begin{aligned}
\left\{\frac{\partial}{\partial t}\right. & \left.+\frac{\mathbf{k}}{m_{\mathrm{e}}} \nabla_{R}-e_{0} \mathbf{E}(t) \nabla_{\mathbf{k}}\right\} f(\mathbf{R}, \mathbf{k}, t) \\
& +\frac{2}{\hbar} \int \mathrm{d}^{3} r \int \frac{\mathrm{d}^{3} k_{1}}{(2 \pi \hbar)^{3}} \sin \frac{\mathbf{r}\left(\mathbf{k}-\mathbf{k}_{1}\right)}{\hbar} V_{I}^{\text {eff }}\left(\mathbf{R}+\frac{\mathbf{r}}{2}, t\right) f\left(\mathbf{R}, \mathbf{k}_{1}, t\right)=I(\mathbf{R}, \mathbf{k}, t),
\end{aligned}
$$

where $I(\mathbf{R}, \mathbf{k}, t) \equiv-2 \operatorname{Re}\left[\tilde{I}^{<}(\mathbf{R}, \mathbf{k}, t, t)\right]=-I_{+}^{<}(\mathbf{R}, \mathbf{k}, t, t)$.

Further, to compute the propagators (retarded and advanced Green's functions) and the spectral function, we consider the sum of the two equations $(129,131)$, i.e. the upper sign in equation (138). Using again a parabolic dispersion, we obtain

$$
\left\{\mathrm{i} \hbar \frac{\partial}{\partial \tau}+\mathrm{i} \hbar \frac{\mathbf{K}_{+}^{A}\left(t_{1}, t_{1}^{\prime}\right)}{t_{1}-t_{1}^{\prime}} \nabla_{\mathbf{k}}-\frac{1}{2 m_{\mathrm{e}}}\left[\left(\frac{\hbar}{2 \mathrm{i}} \nabla_{\mathbf{R}}\right)^{2}+k^{2}+\frac{\left(\mathbf{K}^{A}\right)^{2}\left(t_{1}, t_{1}^{\prime}\right)+\left(\mathbf{K}^{A}\right)^{2}\left(t_{1}^{\prime}, t_{1}\right)}{2}\right.\right.
$$




$$
\begin{aligned}
& \left.\left.+2 \mathbf{k} \mathbf{K}_{+}^{A}\left(t_{1}, t_{1}^{\prime}\right)+\frac{\hbar}{\mathrm{i}} \mathbf{K}_{-}^{A}\left(t_{1}, t_{1}^{\prime}\right) \nabla_{\mathbf{R}}\right]\right\} \tilde{g}^{\gtrless}\left(\mathbf{R}, \mathbf{k}, t_{1}, t_{1}^{\prime}\right) \\
& -\int \mathrm{d}^{3} r \int \frac{\mathrm{d}^{3} k_{1}}{(2 \pi \hbar)^{3}}\left\{\mathrm{e}^{-\frac{\mathrm{i}}{\hbar} \mathbf{r}\left(\mathbf{k}-\mathbf{k}_{1}\right)} V_{I}^{\mathrm{eff}}\left(\mathbf{R}+\frac{\mathbf{r}}{2}, t_{1}\right)+\mathrm{e}^{\frac{\mathrm{i}}{\hbar} \mathbf{r}\left(\mathbf{k}-\mathbf{k}_{1}\right)} V_{I}^{\text {eff }}\left(\mathbf{R}-\frac{\mathbf{r}}{2}, t_{1}^{\prime}\right)\right\} \\
& \times \tilde{g}^{\gtrless}\left(\mathbf{R}, \mathbf{k}_{1}, t_{1}, t_{1}^{\prime}\right)=I_{-}^{\gtrless}\left(\mathbf{R}, \mathbf{k}, t_{1}, t_{1}^{\prime}\right) .
\end{aligned}
$$

What is left in order to obtain the equation for $g^{R / A}$ is to subtract the two equations (142) for $g^{>}$and $g^{<}$, and to add the singular term $\delta\left(t_{1}-t_{1}^{\prime}\right)$ on the right and hand side, cf. definition (54),

$$
\begin{aligned}
& \left\{\mathrm{i} \hbar \frac{\partial}{\partial \tau}+\mathrm{i} \hbar \frac{\mathbf{K}_{+}^{A}\left(t_{1}, t_{1}^{\prime}\right)}{t_{1}-t_{1}^{\prime}} \nabla_{\mathbf{k}}-\frac{1}{2 m_{\mathrm{e}}}\left[\left(\frac{\hbar}{2 \mathrm{i}} \nabla_{\mathbf{R}}\right)^{2}+k^{2}+\frac{\left(\mathbf{K}^{A}\right)^{2}\left(t_{1}, t_{1}^{\prime}\right)+\left(\mathbf{K}^{A}\right)^{2}\left(t_{1}^{\prime}, t_{1}\right)}{2}\right.\right. \\
& \left.\left.\quad+2 \mathbf{k} \mathbf{K}_{+}^{A}\left(t_{1}, t_{1}^{\prime}\right)+\frac{\hbar}{\mathrm{i}} \mathbf{K}_{-}^{A}\left(t_{1}, t_{1}^{\prime}\right) \nabla_{\mathbf{R}}\right]\right\} \tilde{g}^{R / A}\left(\mathbf{R}, \mathbf{k}, t_{1}, t_{1}^{\prime}\right) \\
& \quad-\int \mathrm{d}^{3} r \int \frac{\mathrm{d}^{3} k_{1}}{(2 \pi \hbar)^{3}}\left\{\mathrm{e}^{-\frac{i}{\hbar} \mathbf{r}\left(\mathbf{k}-\mathbf{k}_{1}\right)} V_{I}^{\mathrm{eff}}\left(\mathbf{R}+\frac{\mathbf{r}}{2}, t_{1}\right)+\mathrm{e}^{\frac{i}{\hbar} \mathbf{r}\left(\mathbf{k}-\mathbf{k}_{1}\right)} V_{I}^{\text {eff }}\left(\mathbf{R}-\frac{\mathbf{r}}{2}, t_{1}^{\prime}\right)\right\} \\
& \quad \times \tilde{g}^{R / A}\left(\mathbf{R}, \mathbf{k}_{1}, t_{1}, t_{1}^{\prime}\right)=I_{-}^{R / A}\left(\mathbf{R}, \mathbf{k}, t_{1}, t_{1}^{\prime}\right),
\end{aligned}
$$

where the definition of $I_{-}^{R / A}$ is analogous to that of $I_{-}^{\gtrless}$.

\section{Acknowledgements}

MB is grateful to the Physics Department of the University of Florida, Gainesville, where the bulk of this work has been performed, for its hospitality. We acknowledge discussions with Thomas Fennel (Rostock). This work has been supported by the Deutsche Forschungsgemeinschaft via SFB 198/B10, the National Science Foundation and the Department of Energy (grants DE FG03-98DP00218 and DE FG02ER54677). 


\section{References}

1. Kraeft W.D., Kremp D., Ebeling W., Röpke G. Quantum Statistics of Charged Particle Systems. Berlin, Akademieverlag, 1986.

2. Bonitz M. Quantum Kinetic Theory. Stuttgart/Leipzig, Teubner, 1998.

3. Kadanoff L.P., Baym G. Quantum Statistical Mechanics, 2nd ed. Addison-Wesley, 1989.

4. Klimontovich Yu.L. Kinetic Theory of Nonideal Gases and Nonideal Plasmas. Oxford, Pergamon Press, 1982, (Russian edition: Moscow, Nauka, 1975).

5. Klimontovich Yu.L. Statistical Physics. Chur, Harwood Acad. Publ., 1986, (Russian edition: Moscow, Nauka, 1982).

6. Haug H., Jauho A.P. Quantum Kinetics in Transport and Optics of Semiconductors. New York, Springer-Verlag, Heidelberg, 1996.

7. S. Fujita Introduction to Nonequilibrium Quantum Statistical Mechanics Philadelphia, London, W.B. Saunders Company, 1966.

8. Kremp D., Bornath Th., Bonitz M., Schlanges M. // Phys. Rev. E, 1999, vol. 60, p. 4725 .

9. Bonitz M., Bornath Th., Kremp D., Schlanges M., Kraeft W.D. // Contrib. Plasma Phys., 1999, vol. 39, p. 329.

10. Bonitz M., Dufty J.W. // Superlattices and Microstructures, 2004, vol. 34, p. 225.

11. Bonitz M., Dufty J.W., to be published

12. Mittelman M.H. Introduction to the theory of laser-atom interactions. New York, Plenum Press, 1982.

13. Brack M. // Rev. Mod. Phys., 1993, vol. 65, p. 677.

14. de Heer W.A. // Rev. Mod. Phys., 1993, vol. 65, p. 611.

15. Ichimaru S., Plasma Physics, Benjamin/Cummings Publ. Co. Inc. 1986

16. Salpeter E.E. // Aust. J. Phys., 1954, vol. 7, p. 373.

17. Pines D. Elementary Excitations in Solids. New York, Benjamin, 1963.

18. Bertsch G.F., Yabana K. Density Functional Theory. Introduction to computational methods for many-body systems, (ed. Bonitz M.). Princeton, Rinton Press, to be published.

19. Dirac P.A.M. // Proc. Cambridge Philos. Soc., 1930, vol. 26, p. 376.

20. Semkat D., Kremp D., Bonitz M. // Phys. Rev. E, 1999, vol. 59, p. 1557.

21. Semkat D., Kremp D., Bonitz M. // J. Math. Phys., 2000, vol. 41, p. 7458.

22. Bonitz M. et al. // J. Phys. Cond. Matt., 1996, vol. 8, p. 6075.

23. Bonitz M., Dufty J.W., submitted for publication

24. Kleinert H., Path Integrals in Quantum Mechanics, Statistics and Polymer Physics. World Scientific, Second edition, 1995.

25. Ferry D.K., Zhou J.-R. // Phys. Rev. B, 1993, vol. 48, p. 7944.

26. Vasileska D., Akis R., Knezevic I., Milicic S.N., Ahmed S.S., Ferry D.K. // Microlectronic Engineering, 2002, vol. 63, p. 233.

27. Domps A., Reinhard P.-G., Suraud E. // Ann. Phys. (N.Y.), 2000, vol. 280, p. 211.

28. Calvayrac F., Reinhard P.-G., Suraud E., Ullrich C.A. // Phys. Rep., 2000, vol. 337, p. 493.

29. Kwong N.H., Bonitz M. // Phys. Rev. Lett., 2000, vol. 84, p. 1768.

30. Schäfer W., M. Wegener, Semiconductor Optics and Transport Phenomena, Springer 2002 
31. Bonitz M., Semkat D., Haug H. // Europ. Phys. J. B, 1999, vol. 9, p. 309.

32. Kwong N.H., Bonitz M., Binder R., Köhler H.S. // Phys. Stat. Sol. B, 1998, vol. 206, p. 197.

33. Lipavsky P., Spicka V., Velicky B. // Phys. Rev. B, 1986, vol. 34, p. 6933.

34. Filinov A., Bonitz M., Ebeling W. // J. Phys. A: Math. Gen., 2003, vol. 36, p. 5957.

35. Filinov A., Golubnychiy V., Bonitz M., Ebeling W., Dufty J.W., accepted for publication in Phys. Rev. E, 2004.

\title{
Квантова кінетична теорія металічних кластерів в потужному електромагнітному полі
}

\author{
М.Боніц ${ }^{1,2}$, Д.В.Дафті ${ }^{2}$ \\ 1 Інститут теоретичної фізики та астрофізики \\ Кілльського університету Крістіана Альбрехта, \\ Лейбніцштрассе 15, 24098 Кілль, Німеччина \\ 2 Фізичний факультет університету Флориди, \\ Гейнсвілл, FL 32611-8440
}

Отримано 30 березня 2004 р.

На основі нерівноважних функцій Гріна розроблено квантову теорію слабонеоднорідної системи заряджених частинок. Отримані результати мають суттєве значення для опису валентних електронів металічних кластерів, а також для замкнутих кулонівських систем, таких як електрони в квантових точках чи ультрахолодні іони в пастках. Зокрема, в даній роботі ми зупиняємося на розгляді металічних кластерів, хоча результати можна безпосередньо узагальнити на інші випадки. Ми розпочинаємо з введення в фізику скорельованих валентних електронів у металічних кластерах в сильних електромагнітних полях. Після короткого огляду моделі “желе” та стандартного методу функціоналу густини для опису основних станів ми зосереджуємося на узагальненні теорії на випадок нерівноважних процесів. 3 цією метою розроблено узагальнену калібрувально-інваріантну кінетичну теорію. Ці результати включають рівняння руху для двочасових кореляційних функцій, рівняння для функцій Вігнера та аналіз спектральної функції. Вводиться поняття ефективного квантового потенціалу, який залишає незмінною локальну форму пропагаторів. Це дає нам змогу отримати явні вирази для спектральної функції електронів при суперпозиції сильного електромагнітного поля та слабонеоднорідного утримуючого потенціалу.

Ключові слова: квантова статистична механіка, заряджені кластери, взаємодія лазер-плазма

PACS: 05.30.-d,36.40.Wa,52.38.-r 
